\title{
Measuring the Significance of Spatiotemporal Co-Occurrences
}

\author{
BERKAY AYDIN, AHMET KUCUK, RAFAL A. ANGRYK, and PETRUS C. MARTENS, \\ Georgia State University
}

\begin{abstract}
Spatiotemporal co-occurrences are the appearances of spatial and temporal overlap relationships among trajectory-based spatiotemporal instances with region-based geometric representations. Assessing the significance of spatiotemporal co-occurrences plays an important role in the spatiotemporal frequent pattern mining applications of moving region objects. A spatiotemporal version of the popular Jaccard measure has been used for measuring the strength of spatiotemporal co-occurrences. We will demonstrate the shortcomings of the Jaccard $(J)$ measure when it is used for assessing the significance of co-occurrences among spatiotemporal instances with highly different spatiotemporal evolution characteristics. We will present two extended novel measures $\left(J^{+}\right.$and $\left.J^{*}\right)$ that address the problems linked to the $J$ measure. Our work includes algorithms for the significance measure calculations, the proofs and explanations about the key properties of measures, and a detailed experimental evaluation section. Our experiments include in-depth relevancy and running time analyses demonstrating the suitability of our proposed measures for spatiotemporal frequent pattern mining algorithms.
\end{abstract}

CCS Concepts: • Information systems $\rightarrow$ Spatial-temporal systems; Association rules; • Computing methodologies $\rightarrow$ Spatial and physical reasoning;

Additional Key Words and Phrases: Spatiotemporal knowledge discovery, spatiotemporal objective measure, spatiotemporal co-occurrence patterns

\section{ACM Reference format:}

Berkay Aydin, Ahmet Kucuk, Rafal A. Angryk, and Petrus C. Martens. 2017. Measuring the Significance of Spatiotemporal Co-Occurrences. ACM Trans. Spatial Algorithms Syst. 3, 3, Article 9 (November 2017), 35 pages. https://doi.org/10.1145/3139351

\section{INTRODUCTION}

An important aspect of data mining research is the determination of the interestingness of patterns. In classical frequent pattern mining tasks (e.g., shopping basket analysis), the main goal is to

\footnotetext{
This project has been supported in part by funding from the Division of Advanced Cyberinfrastructure within the Directorate for Computer and Information Science and Engineering, the Division of Astronomical Sciences within the Directorate for Mathematical and Physical Sciences, and the Division of Atmospheric and Geospace Sciences within the Directorate for Geosciences, under the National Science Foundation under Grant No. AC1443061. It was also supported in part by funding from the Heliophysics Living With a Star Science Program, under the National Aeronautics and Space Administrationnder Grant No. NNX15AF39G.

Authors' addresses: B. Aydin, A. Kucuk, and R. A. Angryk, 25 Park Pl NE, 7th Floor, Atlanta, GA 30303; emails: \{baydin2, akucuk1, rangryk\}@cs.gsu.edu; P. C. Martens, 25 Park Pl NE, 6th Floor, Atlanta, GA 30303; email: martens@astro.gsu.edu. Permission to make digital or hard copies of all or part of this work for personal or classroom use is granted without fee provided that copies are not made or distributed for profit or commercial advantage and that copies bear this notice and the full citation on the first page. Copyrights for components of this work owned by others than ACM must be honored. Abstracting with credit is permitted. To copy otherwise, or republish, to post on servers or to redistribute to lists, requires prior specific permission and/or a fee. Request permissions from permissions@acm.org.

(c) 2017 ACM 2374-0353/2017/11-ART9 \$15.00
}

https://doi.org/10.1145/3139351

ACM Transactions on Spatial Algorithms and Systems, Vol. 3, No. 3, Article 9. Publication date: November 2017. 
identify items frequently appearing together in an itemset. Although it seems trivial, such analyses require an appropriate interestingness measure to assess the strength of relationships among different types of items. Measures, such as support, confidence, correlation, and entropy, have been extensively used in frequent pattern mining $[11,53]$.

Spatial and spatiotemporal extensions of frequent pattern mining present a similar challenge, where the choice of objective measure may lead to the discovery of inadvisable or uninteresting information, depending on the context. Unlike classical frequent pattern mining from binary features, in both spatial and spatiotemporal pattern mining tasks, the spatial or spatiotemporal relationships among items (or instances) are not explicit. Therefore, it is considered necessary to initially transform the implicit spatial and temporal information to a transaction-like embodiment. (See Section 2 for the examples of such transformations in the literature.)

There has been extensive research on understanding and assessing the quality, interestingness, and appropriateness of objective measures for different tasks and domains. However, there is no prevalent agreement on selecting the right measure [21]. Selection of the interestingness measure is of great importance, because many measures create conflicting information due to their significantly different properties [54]. Many have agreed there is no universal solution for interestingness measure selection, because the appropriateness of the measures is dependent on the domain and data mining task [41].

\subsection{Problem Formulation}

In this work, we are interested in measuring the strength of spatiotemporal co-occurrences in the context of spatiotemporal co-occurrence pattern (STCOP) mining. Given a set of event types and their region trajectory-based event instances, STCOP mining discovers subsets of event types whose instances frequently co-occur in both space and time [46]. The co-occurrence relationship among the event instances is characterized by spatial and temporal overlap. The significance of a co-occurrence indicates the strength of the overlap relationship, and it is primarily utilized for filtering the spurious co-occurrences from the genuine ones.

The event instances (denoted as ins) are represented with evolving region trajectories, and every instance has an event type that represents the class of the instance. Evolving region trajectories are moving region objects whose spatial representations continuously evolve over time. An evolving region trajectory is comprised of a chronologically ordered collection of time-geometry pairs $\left(t g p_{i}=\left(t_{i}, g_{i}\right)\right)$. Each time-geometry pair represents the region-based location $\left(g_{i}\right)$ of the instance at a particular time $\left(t_{i}\right)$.

In Figure 1(a) and (b), we illustrate two example spatiotemporal co-occurrences. In Figure 1(a), we demonstrate the co-occurrence between two instances, $i n s_{i}$ and $i n s_{j}$, where their regions spatiotemporally overlap during their entire lifespans. In Figure 1(b), we display three co-occurring instances, $i n s_{i}$, ins $s_{k}$, and $i n s_{l}$. Note that all three instances spatially overlap between $t_{4}$ and $t_{5}$.

In STCOP mining, the event instances are considered as three-dimensional objects (with one temporal and two spatial dimensions) associated with a spatiotemporal volume. The strength (i.e., significance) of a co-occurrence is measured using the co-occurrence coefficient (denoted as cce), and the co-occurrences are considered as significant, only if they pass the user-determined co-occurrence coefficient threshold $\left(c c e_{t h}\right)$. Currently, the $c c e$ is calculated using a spatiotemporal version of faccard $(J)$ or OMAX measures [10,46]. Both of these measures utilize the three-dimensional volumes of the co-occurring instances when assessing the significance of cooccurrences. In Figure 1(c), we demonstrate the three-dimensional modeling and spatiotemporal volume transformation of an event instance. The spatiotemporal volume of an instance is calculated by summing the volumes of tgps during its lifespan (as shown in Equation (1)). The lifespan 


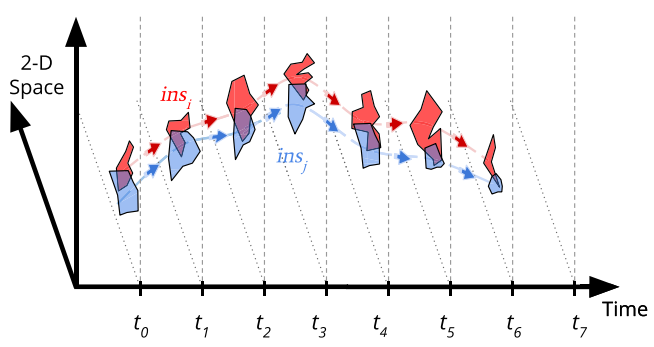

(a)

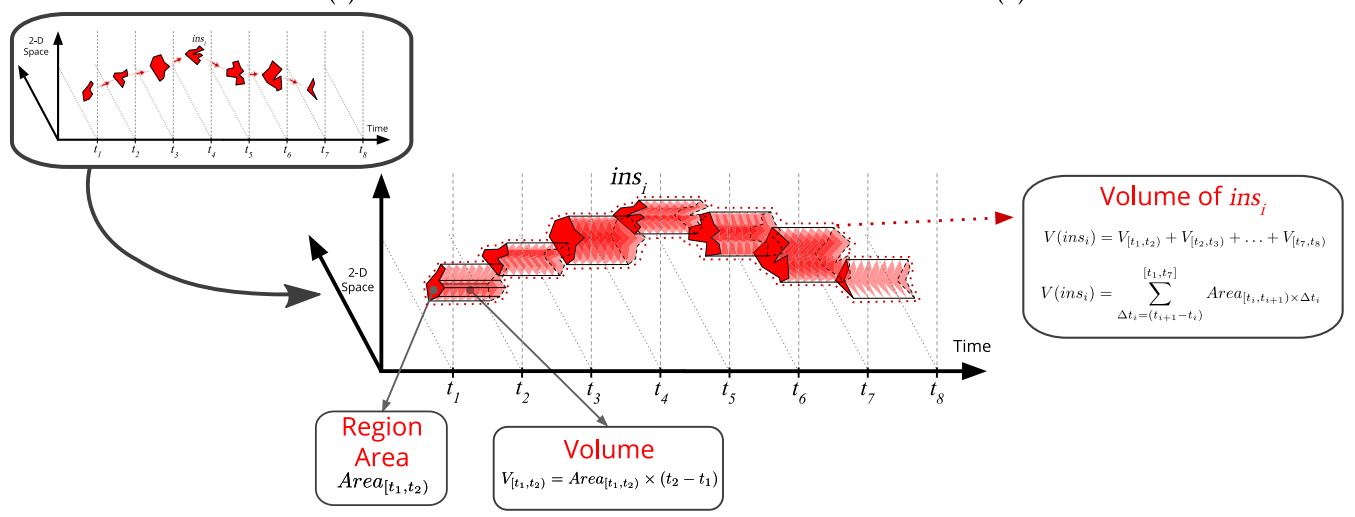

(c)

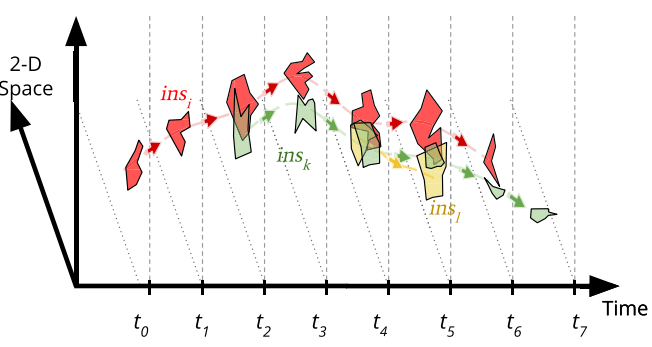

(b)

Fig. 1. Two example spatiotemporal co-occurrences among event instances are shown in (a) and (b). In (a), $i_{i} s_{i}$ co-occurs with ins $j_{j}$. In (b), three instances (ins $s_{i}, i n s_{j}$, and $i n s_{k}$ ) co-occur. In (c), three-dimensional modeling of an event instance is illustrated.

of an instance is the time interval between the start time $\left(t_{s}\right)$ and end time of the instance $\left(t_{e}\right)$.

$$
V\left(\text { ins }_{i}\right)=\sum_{\Delta t_{i}=\left(t_{i+1}-t_{i}\right)}^{\left[t_{s}, t_{e}\right)} \operatorname{Area}_{\left[t_{i}, t_{i+1}\right) \times \Delta t_{i}} .
$$

The volume of an individual time-geometry pair is found by multiplying the area of the region geometry by the duration (the length of the time interval) as shown in Equation (2). Note that, for each time-geometry pair, the volume is discretely calculated.

$$
V_{\left[t_{i}, t_{i+1}\right)}=\operatorname{Area}_{\left[t_{i}, t_{i+1}\right)} \times\left(t_{i+1}-t_{i}\right) .
$$

\subsection{Motivation}

The main focus of this work is to explore and analyze the significance assessment techniques of spatiotemporal co-occurrences. An appropriate significance measure for co-occurrences is necessary for the relevancy of the results, particularly in the STCOP mining. As mentioned earlier, current STCOP mining algorithms utilize the spatiotemporal version of Jaccard $(J)$ and OMAX measures ${ }^{1}$ for determining the strength of co-occurrences $[10,46,47]$. However, using the $J$ or $O M A X$ measure leads to unfair assessments in certain cases, which can cause the exclusion of the important co-occurrences and the inclusion of spurious ones. In this work, we aim to create more

${ }^{1}$ OMAX measure is primarily used for filtering in STCOP mining. The significance of the spatiotemporal co-occurrences is ultimately decided based on the $J$ measure. 
relevant significance measures for spatiotemporal co-occurrences, which can potentially alleviate these issues.

Large-scale analysis of spatiotemporal co-occurrences can help predict relationships among event types and verify the currently known relationships in various scientific fields such as astronomy, meteorology, and geophysics [46]. Space weather prediction is an example application area of STCOP mining. (See Section 3 for more information on the unbalanced spatiotemporal characteristics of solar event data.) Our everyday lives, government, and industry can be impacted by geomagnetic activity of the Sun, as solar storms affect space and air travel, communication satellites, navigation systems, and power grids. A 2008 report published by the National Research Council [52] estimates that the cost of a large, Earth-directed solar superstorm would have a societal and economic impact on the U.S. economy worth between 1 and 2 trillion dollars during the first year alone, with recovery times of 4 to 10 years. Although we highlight the solar event data in our examples, the disproportions of spatiotemporal data are common in nature. For instance, the proliferation and growth of cancer stem cells differs significantly based on the micro-environment in which they reside [60]. Another example is the drastic change of sizes in midget, normal, large, and giant hurricanes and the tropical storms associated with them [31].

\subsection{Contributions and Scope}

In this work, we extend our earlier work [4] and present novel techniques for assessing the significance of spatiotemporal co-occurrences appearing among the spatiotemporal instances. Our contributions include (1) developing two novel and efficient objective measures, $J^{+}$and $J^{*}$, which can be used for assessing spatiotemporal significance instead of the popularly used $J$ measure; (2) presenting algorithms for calculating our new measures; (3) providing theoretical analysis on our measures' key properties, which are antimonotonicity and containment, in order to specify the appropriateness of the measure to the current algorithms [10,46]; and (4) verifying the correctness and applicability of the measures by providing extended experimental results demonstrating the computational efficiency and relevancy of the $J^{+}$and $J^{*}$ measure.

The following issues are beyond the scope of this work: (1) modeling a prevalence measure for the assessment of frequency of spatiotemporal co-occurrence patterns; (2) determining threshold values for spatiotemporal significance of co-occurrences.

\subsection{Outline}

The rest of this article is organized as follows. We present the related work on spatial and spatiotemporal data mining algorithms in Section 2. We continue our discussion with a real-life example from solar event data in Section 3, where we demonstrate our motivation and the disadvantages of using the $J$ measure. In Sections 4 and 5, we explain the $J^{+}$and $J^{*}$ measures, in detail, with algorithms and their important properties. We present our experimental evaluation in Section 6. Finally, in Section 7, we conclude the article after presenting possible future directions.

\section{RELATED WORK ON SPATIAL AND SPATIOTEMPORAL DATA MINING}

Below, we will present the related spatial and spatiotemporal data mining studies. In these studies, the implicit spatial and temporal close by relationships are translated into composite transactionlike structures (e.g., co-locations, flocks, episodes, co-occurrences). Although the mining subject of these studies is usually distinct for each study, all of them focus on the spatial or spatiotemporal closeness of objects. We will primarily explore how they formalize the generic close by relationships in their respective studies. 


\subsection{Spatial Co-Locations}

Spatial association rules are association rules involving spatial relations among spatial objects [33]. Kopersky and Han introduced a reference feature centric model for discovering spatial association rules. In the reference feature centric model, one or more user-specified reference features are selected and transactions are created based on the spatial proximity of the instances to the reference points. The spatial proximity is defined as the generic close to (g_close_to) relationship, which conceptually includes topological relations such as intersection, inside, or close by.

The spatial co-location patterns (or neighboring class sets) represent the subsets of features whose instances are frequently located together [27, 28, 42, 59]. To find the co-located objects, Morimoto introduced a space-driven partitioning strategy [42]. In this strategy, the space is divided into disjoint partitions and spatial instances are considered as a co-location only if they are located in the same space partition. Later, Huang et al. presented an event-centric neighborhooding strategy for co-location pattern mining [28]. The neighborhooding strategy can capture the spatial neighborhoods without specifically determining the reference features or partitioning the space. The possible criteria for forming neighborhoods include spatial (or spatiotemporal) relationships (adjacency, overlap), metric relationships (distance-based approaches), or a combination of these two. Later, Huang et al. proposed algorithms for mining co-location patterns with rare events in [27]. This work primarily focuses on discovering the co-location patterns containing rarely occurring phenomena (with high confidence) using the maximal participation ratio ( $\max P R$ ) measure. The $\max P R$ measure denotes the maximum relative frequency of the event types in a particular co-location measure. While there are sufficiently great differences between the interestingness of spatial co-location patterns and the significance of spatiotemporal co-occurrences, the proposed approach can remedy some of the problems associated to $J$ measure. We will provide a detailed discussion on the similarity of mining co-locations with rare events and measuring the significance of spatiotemporal co-occurrences in Section 3. In addition, Xiong et al. used a buffer-based event-centric neighborhooding approach for identifying the co-locations of spatial instances with extended geometric representations [59]. In the buffer-based model, given a distance $d$ for forming a buffer, the spatial instances are considered as co-located when their buffers spatially overlap.

Yoo et al. introduced the discovery of co-evolving spatial event sets in [61]. Co-evolving spatial event sets represent the co-location patterns whose prevalence values similarly evolve over time. In this work, the distance-based event-centric neighborhooding strategy (from [28]) is used to find co-location patterns. The prevalence of the spatial co-location patterns are measured by the participation index. Participation index is the minimum relative frequency of the participating event types in a pattern. Then, a spatial prevalence time sequence, which is comprised of a sequence of participation index values, is generated for each co-location pattern. To find the similarity between two spatial prevalence time sequences, normalized Euclidean distance is used.

Mining collocation episodes is introduced in [14]. A collocation episode is defined for pointbased objects, and it is a sequence of spatial co-location relationships, each describing which pairs of object types are close to each other over a significant time window. The closeness of the colocation sequences is determined using an aggregate distance function defined as either maximum or average pairwise distances between the point-based objects.

\subsection{Moving Cluster Analysis}

The Relative Motion (REMO) framework was developed by Laube and Imfeld to discover the motion patterns in groups of spatiotemporal trajectories [35]. The framework builds a REMO analysis matrix from the point-based spatiotemporal trajectories using motion attributes (i.e., speed, change of speed, and motion azimuth). Later, the REMO analysis matrix is analyzed to find the 
motion patterns such as constance, concurrence, and propagation. For example, the concurrence pattern represents a group of objects showing synchronous motion at a time interval.

This work was extended to include the motion patterns using the spatial neighborhood information in $[22,36]$. In other words, the motion patterns are spatially constrained based on their closeness and complex spatiotemporal patterns such as track, flock, leadership, encounter, and convergence are generated. Gudmundsson et al. formalized the spatial proximity using a circular impact range [22]. For example, the flock pattern is the spatially constrained version of the concurrence pattern. Namely, a set of objects is considered to form a flock if they are within a circular region (of radius $r$ ) and they move in the same direction. Apart from the impact range approach, Laube et al. listed three more alternative approaches for spatial proximity constraints: (1) maximal length of cumulated distances to the mean or median center of the objects; (2) average length of the Delaunay edges of a group forming a relative motion pattern; and (3) maximal border length of the convex hull formed by a group of objects.

Kalnis et al. defined the problem of discovering moving clusters, and proposed clustering-based methods to identify moving clusters [30]. For finding spatial clusters (i.e., objects that are spatially close by at a particular time) in each spatial snapshot, they use the density-based spatial clustering algorithm, DBSCAN [18]. If there is a large enough number of common spatial objects between two clusters in consecutive time slots, such clusters are called moving clusters. The portion of common objects between two consecutive clusters is measured by a Jaccard-like integrity measure $-\frac{\left|c_{t} \cap c_{t+1}\right|}{\left|c_{t} \cup c_{t+1}\right|}$, where $c_{t}$ and $c_{t+1}$ denote two consecutive clusters (set of objects) at times $t$ and $t+1$.

\subsection{Spatiotemporal Co-Occurrences}

Celik et al. introduced the mixed-drove spatiotemporal co-occurrence patterns (MDCOPs) in [16]. Similar to Huang et al.'s work on co-location patterns [28], Celik et al. used the distance-based event-centric neighborhooding approach to generate spatiotemporal neighborhoods when mining MDCOPs [16]. In MDCOP mining, the time frames are collapsed, meaning the temporal framework is divided into disjoint time frames. For each time frame (1) the event instances are considered to be in temporal neighborhood and (2) the prevalent spatial co-locations, which occur during the same time frame are found. Then, MDCOPs, which can be interpreted as temporally persistent spatial colocation patterns, are determined by checking their temporal persistence (time prevalence). In [16], the time prevalence is measured as the ratio of time frames where a co-location pattern is present to the total number of time frames. In Celik's succeeding work [15], the discovery of partial spatiotemporal co-occurrence patterns (PACOPs) are inspected. PACOPs are very similar to MDCOPs. These two works differ in finding the time prevalence of co-occurrence patterns. When finding PACOPs, the algorithm considers the partially present (i.e., less frequently occurring) object types, and uses the temporal participation index when determining the time prevalence. MDCOP mining uses a support-like time prevalence measure, which is based on the frequency, while PACOP mining uses the temporal participation index, which is based on the relative participation (frequency).

Pillai et al. introduced STCOPs and STCORs from datasets with evolving regions [46] and [48]. Recently, spatiotemporal event sequence (STES) mining algorithms also make use of the spatiotemporal co-occurrence relationship among the evolving regions [6-8]. Similar to the approach in [59], event instances are considered to form a spatiotemporal co-occurrence if there exists a spatiotemporal overlap among these instances. In contrast to Xiong's approach, a buffer is not used and a spatiotemporal version of Jaccard $(J)$ measure is employed for measuring the significance of the co-occurrences. In addition to the $J$ measure, overlap measures OMIN (Overlap Minimum) and $O M A X$ (Overlap Maximum) and spatiotemporal versions of Dice, Cosine measures are also used as a filter to the $J$ measure. In [6-8], the $J^{*}$ measure is used for the significance assessments. 
2.3.1 Antimonotonic Property. A prevalent STCOP is a set of event types, whose event instances frequently and strongly co-occur together in both space and time. The prevalence of STCOPs is measured using the participation index, and shows how interesting a pattern is by demonstrating information about the relative frequency of participating instances to the total number of instances of a particular event type in a dataset. The prevalence of STCOPs is measured on event type level. On the other hand, the strength of spatiotemporal co-occurrences is measured on instance level, and implies how significant a co-occurrence is. Readers can refer to the Online Appendix (Part A) of this article for the details on the STCOP mining algorithm [46].

Similar to the spatial co-location and spatiotemporal co-occurrence pattern mining algorithms in this section [14-16, 28, 59], STCOP mining algorithms are Apriori based [10, 46, 47]. The spatiotemporal co-occurrences are considered significant if they can pass a given co-occurrence coefficient threshold. Apriori-based mining algorithms are heavily dependent on the downward closure property (i.e., antimonotonicity) of the prevalence and significance measures. The antimonotonicity property enables the frequent pattern mining algorithms to efficiently prune the search space [25]. Given a co-occurrence coefficient threshold, antimonotonicity for a particular spatiotemporal co-occurrence guarantees that if the co-occurrence is significant (based on the given threshold), then all the co-occurrences appearing among the subsets of participating instances are also significant.

The currently used significance measures $(J$ and OMAX) for STCOP mining carry the antimonotonic property. In Sections 4 and 5, we will discuss the antimonotonic property of $J^{+}$and $J^{*}$ measures in detail.

\subsection{Summary}

Although our work is related to spatial co-location mining and discovery of moving clusters, it is primarily associated with STCOP mining. We demonstrate a summary of related studies on spatial and spatiotemporal data mining in Table 1. Previous works in spatiotemporal co-occurrence pattern mining can be classified into two categories based on the data types: (1) patterns discovered from point-based spatiotemporal event instances such as MDCOPs or PACOPs [15, 16]; and (2) patterns discovered from region-based spatiotemporal event instances such as STCOPs or STCORs $[46,48]$. Our proposed significance measures are designated for region-based spatiotemporal event instances that form trajectories over time.

\section{A REAL-LIFE EXAMPLE}

Spatiotemporal co-occurrences commonly occur among various types of solar events (or features) such as Active Regions (AR), Coronal Holes (CH), Emerging Flux (EF), Filaments (FI), Flares (FL), Sigmoids (SG), and Sunspots (SS) [5]. The spatial characteristics such as location, shape, and size of solar event instances continuously evolve over time. Figure 2 demonstrates the spatiotemporal evolution of two co-occurring solar events (one AR and one SS). The regions covered by these solar events are represented as polygons evolving over time. Therefore, the solar events can be modeled as event instances formed by evolving region trajectories.

As mentioned earlier, spatiotemporal co-occurrences appear when there is a spatiotemporal overlap (being at the same location and at the same time) of two or more event instances, and their significance is calculated using the co-occurrence coefficient (cce). The cce is calculated as the $J, O M I N$, and $O M A X$ measures $[46,48]$. The $J$ measure (shown in Equation (3)) is the ratio of intersection volume to the union volume of two or more overlapping instances.

$$
J\left(i n s_{1}, \ldots, i n s_{n}\right)=\frac{V\left(i n s_{1} \cap \ldots \cap i n s_{n}\right)}{V\left(i n s_{1} \cup \ldots \cup i n s_{n}\right)} .
$$


Table 1. Summary of Related Work on Spatial and Spatiotemporal Data Mining

\begin{tabular}{|c|c|c|c|c|c|}
\hline Pattern Type & Spatial & Temporal & Strategy & Data Type & References \\
\hline $\begin{array}{r}\text { Spatial Association } \\
\text { Rules }\end{array}$ & $\sqrt{ }$ & $x$ & $\begin{array}{c}\text { Reference feature } \\
\text { selection with a generic } \\
\text { close to relation }\end{array}$ & $\begin{array}{l}\text { Point or } \\
\text { Region }\end{array}$ & [33] \\
\hline Neighboring Class Sets & $\sqrt{ }$ & $x$ & Disjoint spatial partitions & Point & {$[42]$} \\
\hline Spatial Co-locations - 1 & $\sqrt{ }$ & $x$ & $\begin{array}{l}\text { Event-centric approach } \\
\text { based on distance }\end{array}$ & Point & [28], [27] \\
\hline Spatial Co-locations - 2 & $\sqrt{ }$ & $x$ & $\begin{array}{l}\text { Event-centric approach } \\
\text { based on buffer }\end{array}$ & $\begin{array}{l}\text { Point or } \\
\text { Region }\end{array}$ & {$[59]$} \\
\hline $\begin{array}{r}\text { Co-evolving spatial } \\
\text { event sets }\end{array}$ & $\sqrt{ }$ & $\sqrt{ }$ & $\begin{array}{l}\text { Event-centric approach } \\
\text { based on distance and } \\
\text { normalized Euclidean for } \\
\text { time sequences }\end{array}$ & Point & [61] \\
\hline Collocation Episodes & $\sqrt{ }$ & $\sqrt{ }$ & $\begin{array}{l}\text { Aggregate distance } \\
\text { function (avg. or max. } \\
\text { pairwise distance } \\
\text { between points) }\end{array}$ & Point & [14] \\
\hline $\begin{array}{l}\text { Relative Motion } \\
\text { Patterns (Flock, } \\
\text { Convergence, } \\
\text { Leadership, etc.) }\end{array}$ & $\sqrt{ }$ & $\sqrt{ }$ & $\begin{array}{l}\text { Relative motion } \\
\text { parameters based on the } \\
\text { pattern type and spatial } \\
\text { proximity constraints } \\
\text { (impact range, cumulated } \\
\text { distance, Delaunay edges, } \\
\text { length of convex hull) }\end{array}$ & Point & [35], [22], [36] \\
\hline Moving Clusters & $\sqrt{ }$ & $\sqrt{ }$ & $\begin{array}{l}\text { Density-based spatial } \\
\text { clustering and integrity } \\
\text { threshold }\end{array}$ & Point & {$[30]$} \\
\hline $\begin{array}{r}\text { Mixed-drove } \\
\text { spatiotemporal } \\
\text { co-occurrence patterns }\end{array}$ & $\sqrt{ }$ & $\sqrt{ }$ & $\begin{array}{c}\text { Spatial participation } \\
\text { index in collapsed time } \\
\text { frames and time } \\
\text { prevalence (support-like) }\end{array}$ & Point & {$[16]$} \\
\hline $\begin{array}{l}\text { Partial spatiotemporal } \\
\text { co-occurrence patterns }\end{array}$ & $\sqrt{ }$ & $\sqrt{ }$ & $\begin{array}{l}\text { Spatial participation } \\
\text { index in collapsed time } \\
\text { frames and temporal } \\
\text { participation index based } \\
\text { on relative frequency }\end{array}$ & Point & {$[15]$} \\
\hline $\begin{array}{r}\text { Spatiotemporal } \\
\text { co-occurrence patterns } \\
\text { and rules (STCOP - } \\
\text { STCOR), and event } \\
\text { sequences (STES) from } \\
\text { evolving regions }\end{array}$ & $\sqrt{ }$ & $\sqrt{ }$ & $\begin{array}{l}\text { Measure the strength of } \\
\text { spatiotemporal overlap } \\
\text { using the Jaccard } \\
\text { measure }\end{array}$ & Region & $\begin{array}{c}{[46],[48],[8],} \\
{[7],[6]}\end{array}$ \\
\hline
\end{tabular}

The intersection volume is calculated from the areas of intersecting regions at times where they spatiotemporally overlap, while the union volume is calculated by spatially unionizing the region geometries for all valid time intervals.

The overlap measures OMIN and OMAX (shown in Equations (4) and (5)) are calculated as the ratio of intersection volume to the maximum and minimum volume of the instances.

$$
\operatorname{OMAX}\left(i n s_{1}, \ldots, i n s_{n}\right)=\frac{V\left(i n s_{1} \cap \ldots \cap i n s_{n}\right)}{\max \left(i n s_{1}, \ldots, i n s_{n}\right)}
$$



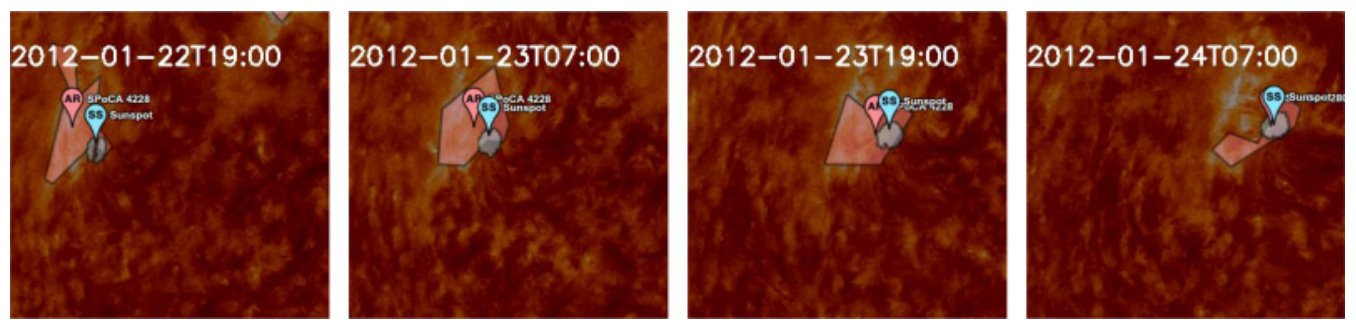

Fig. 2. Polygon representations of two co-occurring solar event instances (an active region and a sunspot) reported by Heliophysics Event Knowledgebase [37] between "22 January 2012 19:00" and "24 January 2012 07:00." These events are tracked by the algorithm presented in [32].

$$
\operatorname{OMIN}\left(i n s_{1}, \ldots, \text { ins }_{n}\right)=\frac{V\left(i n s_{1} \cap \ldots \cap i n s_{n}\right)}{\min \left(i n s_{1}, \ldots, i n s_{n}\right)} .
$$

The $O M I N, O M A X$, and $J$ measures output a value on $[0,1]$ range. The value 0 means there is no co-occurrence, and 1 means the co-occurring trajectories are equal. ${ }^{2,3}$

Figure 3 demonstrates the histograms of area, lifespan, and volume of seven different solar event types registered by NASA's Solar Dynamic Observatory telescope, and reported by the Heliophysics Event Knowledgebase [29]. The area refers to the region's area for individual timegeometry pairs, while the lifespan refers to the time duration between start and end times of the instances.

It can be observed from Figure 3 that lifespans, volumes, and areas of the solar event instances exhibit drastic variability. In Figure 3, the horizontal axes are in logarithmic scale and shared across the rows in the same column. Events such as sunspots and filaments have very long lifespans, while flares, sigmoids, and emerging flux events have very short ones. On the other hand, the volumes of coronal holes and active regions are very large compared to flares and emerging flux events. The spatiotemporal co-occurrence of large volume event instances with smaller ones leads to very large union volumes. However, the intersection volume is limited to the volume of the smaller event (see the $J$ and $O M A X$ measure in Equation (3)). Furthermore, the events with longer lifespans and larger areas have higher chances of co-occurring with other events. These situations breed anomalies when assessing the strength of spatiotemporal co-occurrences.

Consider the following example scenarios to see the need for a different approach when calculating the co-occurrence coefficient in spatiotemporal co-occurrence pattern mining.

Example 1-Coverage anomaly: In Figure 4(a), two event instances (ins $s_{A}$ and $i n s_{B}$ ) are demonstrated. $i n s_{A}$ has small area and shorter lifespan, and $i n s_{B}$ has larger area and longer lifespan. Since ins $s_{A}$ completely covers $i n s_{B}$, we cannot have a stronger spatiotemporal overlap given the state of those two instances. However, the $J$ or $O M A X$ values for $i n s_{A}$ and $i n s_{B}$ are unfairly affected by the large union volume caused by $i n s_{B}$, even though ins $A$ strongly overlaps with $i n s_{B}$ throughout its entire lifespan.

Example 2-Large volume bias: In Figure 4(b), two event instances (ins $C$ and ins $_{D}$ ) are depicted. $i n s_{C}$ and $i n s_{D}$ have both large areas and long lifespans. Their region geometries slightly overlap only at three timestamps, which is a weaker co-occurrence when compared to the examples in Figures 4(a) and (c). Even though their spatiotemporal co-occurrence is limited to a small portion of their longer lifespan, the $J$ or $O M A X$ values for these two instances will tend to be

\footnotetext{
${ }^{2}$ The equality of trajectories means that all of the time-geometry pairs in trajectories are equal.

${ }^{3}$ If an instance is completely covered by another one, the OMIN outputs 1 .
} 

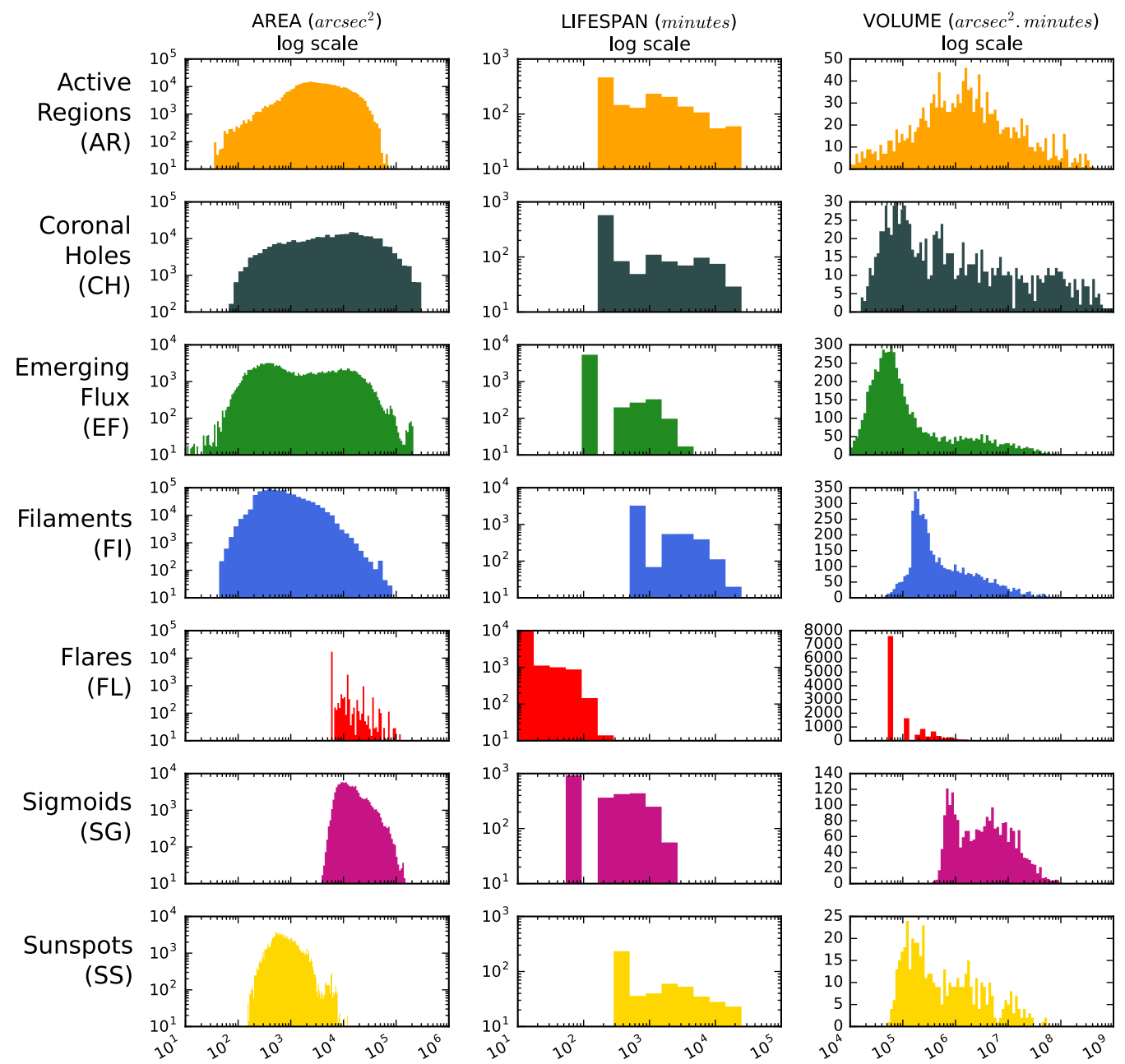

Fig. 3. Histograms of area, lifespan, and volume for seven different types of solar event instances occurred between January 1, 2012 and December 31, 2012.

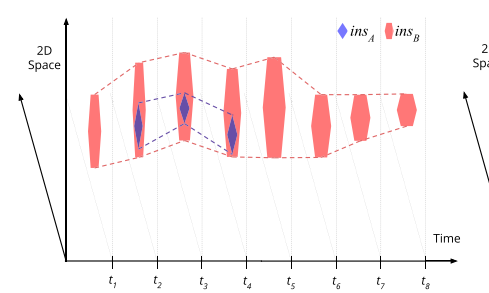

(a)

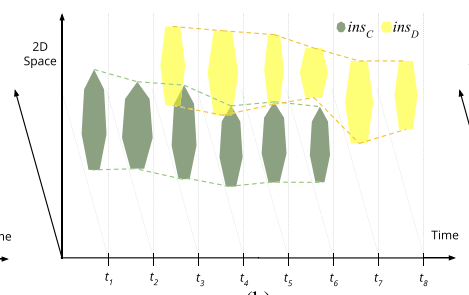

(b)

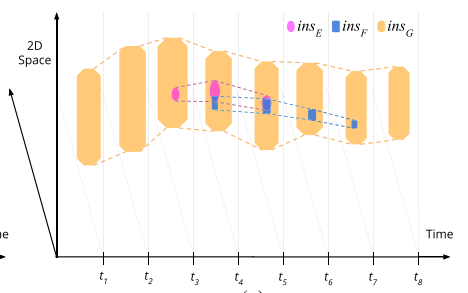

(c)

Fig. 4. The illustration of three possible scenarios for spatiotemporal co-occurrences of instances that can occur among event instances with unbalanced characteristics. 
larger for two reasons: (1) in a fixed spatial and temporal window, instances with larger areas or longer lifespans have higher chances of spatiotemporal overlap, and (2) J and OMAX values tend to go higher as the intersection volume of these two instances is likely higher.

Example 3-Favoring the similar: In Figure 4(c), three event instances (ins $s_{E}$, ins $s_{F}$, and ins $_{G}$ ) are demonstrated. $i n s_{E}$ and $i n s_{F}$ have both small areas and relatively shorter lifespans. On the other hand, $i n s_{G}$ has moderate area but longer lifespan. For the co-occurrence of $i n s_{E}$ and $i n s_{F}$, the $J$ value is higher as their union volume is not high. However, for the co-occurrence of $i n s_{E}$, ins $s_{F}$, and $i n s_{G}$, the $J$ value stays small because of the larger union volume caused by $i n s_{G}$. Similarly, the $O M A X$ value is also small because of the large volume of $i n s_{G}$. Nevertheless, both ins $_{E}$ and $i n s_{F}$ strongly co-occur with ins $_{G}$. A similar problem is also present for Example 2; the instances with similar spatiotemporal characteristics are unfairly favored by the $J$ or OMAX measures especially when a fixed threshold is used for pruning supposedly unimportant co-occurrences.

It can be seen that the $J$ and $O M A X$ measures tend to favor the event instances with similar characteristics. Small volume instances have higher chances of having a strong co-occurrence with each other. Similarly, large volume instances will have higher chances of having a strong co-occurrence with large volume instances. Given the unbalanced nature of solar data (or more generally, scientific data) and possibly disregarded but important co-occurrences among these instances, it is necessary to develop novel techniques for the significance assessment of co-occurrences.

A Discussion on Similarity with Co-location Patterns with Rare Events: As mentioned earlier in Section 2, mining co-location patterns involving rare event types was proposed in [27]. In this work, Huang et al. proposed a new co-location pattern mining approach primarily targeted for rarely occurring phenomena. Their main goal is to discover interesting co-location patterns involving event types which have substantially different frequency levels. Their approach is specifically designated for the rarely appearing events and their primary contribution is introducing the $\max P R$ measure, which identifies the maximum relative frequency of the event types in spatial co-location patterns.

The participation ratio ( $p r)$ of an event type (in a pattern) measures the ratio of the event instances, which participate in the instances of a pattern [28].

$$
\operatorname{pr}\left(C L P, e_{i}\right)=\frac{\text { Number of unique instances of } e_{i} \text { participated in a pattern instance of } C L P}{\text { Total number of instances of } e_{i}} .
$$

For example, for an event type $e_{i}$, which participates in a co-location pattern, $C L P=\left\{e_{i}, e_{j}\right\}$, the $\operatorname{pr}\left(C L P, e_{i}\right)$ is calculated as the ratio between the number of unique instances of $e_{i}$, which participate in a pattern instance of $C L P$, and the total number of instances of $e_{i}$. Hence, the participation ratio, $\operatorname{pr}\left(C L P, e_{i}\right)$, shows the relative frequency of the events participating in a pattern. Then, given the participation ratios of the event types in a pattern, the $\max P R$ measure is calculated as the maximum participation ratio.

$$
\max P R(C L P)=\max _{e_{k} \in C L P}\left(\operatorname{pr}\left(C L P, e_{k}\right)\right) .
$$

For our example co-location pattern, $\max P R(C L P)$ is calculated as $\max \left(\operatorname{pr}\left(C L P, e_{i}\right), \operatorname{pr}\left(C L P, e_{j}\right)\right)$. It is also important to note that the original spatial co-location pattern mining approach [28] uses participation index ( $p i)$ measure, which is the minimum of the $p r$ values.

$$
p i(C L P)=\min _{e_{k} \in C L P}\left(\operatorname{pr}\left(C L P, e_{k}\right)\right) .
$$

Using the $\max P R$ measure, it is possible to identify the spatial co-locations involving rarely occurring phenomena, because unlike the pi measure, the $p r$ values of frequently occurring events would not decrease the $\max P R$ measure. 
The $\max P R$ measure is motivated with similar reasons as our $J^{*}$ measure, where the pi measure unfairly assesses the interestingness of rarely occurring phenomena. The limitation of pi measure particularly resembles the Coverage Anomaly that we have discussed in Example 1. Even though the participation ratio of the rarely occurring event types are high within the co-location pattern (similar to the intersection volume being maximal in a co-occurrence as in Figure 4(a)), the pi value is calculated as the minimum $p r$. When a co-location pattern involves frequently occurring events, the $p r$ values of frequently occurring events would decrease the $p i$ value of the pattern.

It is also important to point out the differences between the interestingness measures for co-location patterns and the significance measures of spatiotemporal co-occurrence relationships. Firstly, the $\max P R$ and $p i$ measures assess the interestingness of a co-location pattern (or patterns in the form of Boolean features), while our proposed measures assess the significance of the spatiotemporal co-occurrence relationship among trajectory-based event instances. Secondly, the measures proposed for the significance of spatiotemporal co-occurrences operate on the spatiotemporal volumes. On the other hand, the prevalence (interestingness) of spatial co-location patterns is measured using participation index $(p i)$ and maximal participation ratio $(\max P R)$ measures, which count the number of Boolean spatial features. Because of these reasons, we can suggest that $\max P R$ cannot be used for fairly measuring the significance of spatiotemporal co-occurrences in its current form. For example, there are many instances of Flares that are short-lasting and have small volumes (disadvantage in spatiotemporal co-occurrence relationships); however, they are one of the most commonly occurring phenomena in solar event datasets (advantage in spatial co-location patterns).

Another observation about the $\max P R$ and $p i$ measures is that they resemble the overlap measures, OMAX and OMIN. The OMAX measure gets the maximum volume in denominator (in Equation (4)), which fundamentally corresponds to pi measure (in Equation (8)) in the context of spatial co-location pattern mining. The minimum $p r$ value belongs to the most frequently occurring event type (supposing the number of participating events are the same for each event type), and this is analogous to the spatiotemporal trajectory with the largest volume in a spatiotemporal co-occurrence. On the other hand, the $O M I N$ uses the minimum volume (in Equation (5)) and corresponds to the $\max P R$ measure (in Equation (7)). The maximum $p r$ value belongs to the most rarely occurring phenomena, where the total number of events is the smallest (supposing the number of participating events is the same for each event type). We will provide experimental analysis for the overlap measures (OMIN and OMAX) in Section 6, and thoroughly describe the calculation of these measures in our Online Appendix.

\section{EVOLUTION OF SPATIOTEMPORAL JACCARD MEASURE}

\subsection{Preliminaries}

The support (denoted as supp in Equation (9)) measure for an association rule in classical frequent itemset mining is the fraction of transactions that includes all the participating item types (denoted as $I_{i}$ ) in the entire database [1]. Support is usually used for assessing the significance of a pattern or an association rule and it represents the joint probability of two or more item types in a sample dataset.

$$
\operatorname{supp}\left(I_{1}, I_{2}, \ldots, I_{n}\right)=P\left(I_{1} \cap I_{2} \cap \ldots \cap I_{n}\right) .
$$

The Jaccard similarity coefficient has been extensively used for measuring the similarity among item types (in shopping basket analysis) [55], documents (in text mining) [26, 58], or spatial feature types and objects $[10,46,47,56]$. Following the item type representation in Equation (9), the Jaccard 
similarity coefficient (Jaccard) for itemsets is calculated as follows:

$$
\operatorname{Jaccard}\left(I_{1}, I_{2}, \ldots, I_{n}\right)=\frac{P\left(I_{1} \cap I_{2} \cap \ldots \cap I_{n}\right)}{P\left(I_{1} \cup I_{2} \cup \ldots \cup I_{n}\right)} .
$$

The generalized version of Jaccard similarity coefficient (in Equation (10)) can be expressed as Steinhaus index [17]. Given a measurable space, and a measurement function $\mu$, Steinhaus index is defined as follows:

$$
\operatorname{Steinhaus}\left(I_{1}, I_{2}, \ldots, I_{n}\right)=\frac{\mu\left(I_{1} \cap I_{2} \cap \ldots \cap I_{n}\right)}{\mu\left(I_{1} \cup I_{2} \cup \ldots \cup I_{n}\right)} .
$$

For the case of the classical Jaccard similarity coefficient (in Equation (10)), the cardinality of a given sample set is the measurement function. In STCOP mining [46], a spatiotemporal version of Jaccard measure (i.e., the $J$ measure) is used for assessing the strength of a spatiotemporal co-occurrence. The $J$ is a version of Steinhaus index, where the measurement function $(\mu)$ is the volume function $(V)$ presented in Equation (1). In Equation (3), the measurement function, $V$, calculates the intersection and union volumes of trajectory-based event instances.

\subsection{Intermediate Form: $\mathrm{J}^{+}$Measure}

Three problems associated with the $J$ measure are mentioned in Section 3 with example scenarios. One intuitive solution for alleviating the problems addressed in Section 3 is to modify the measurement function $(\mu)$ to eliminate the segments of trajectories when calculating the $c c e$. The criterion for elimination that we employ is the existence of spatiotemporal co-occurrence (i.e., spatiotemporal overlap relationship) among the instances. Using the overlap-based criterion can help us focus on segments of trajectories, where co-occurrences appear.

Let $J^{+}$be an extended version of Jaccard measure. We define $J^{+}$as follows:

$$
J^{+}\left(i n s_{1}, \ldots, i n s_{n}\right)=\frac{V_{t i l} c o\left(i n s_{1} \cap \ldots \cap i n s_{n}\right)}{V_{t i l c o}\left(i n s_{1} \cup \ldots \cup i n s_{n}\right)} .
$$

Here, the measurement function of $J$ (i.e., $V$ in Equation (3)), is replaced by an interval volume function, $V_{t i l^{c o}} . V_{t i l^{c o}}$, which measures the volume of intersection and union at times where there exists a spatiotemporal overlap among all the instances.

Definition 4.1. Interval volume function, $V_{t i l}$, calculates the volume of given trajectory-based geometries only for the time intervals given in a time interval list, denoted as til.

For a given trajectory-based instance ins $s_{i}$, interval volume function is calculated using $V_{t i l}$ (Equation (13)). It calculates the volume of the trajectory-based instance only for the intervals specified in the time interval list $(t i l)$.

$$
V_{t i l}\left(\text { ins }_{i}\right)=\sum_{\left[\tau_{k}, \tau_{k+1}\right) \in t i l} \operatorname{Area}_{\tau_{k}}\left(\text { ins }_{i}\right) \times\left(\tau_{k+1}-\tau_{k}\right) .
$$

Definition 4.2. Time interval list $(t i l)$ is a list of ordered time intervals. Each time interval is defined by a pair of timestamp values $\left(t_{i}, t_{j}\right)$, where $t_{i}<t_{j}$; for each $i, j ; 1 \leq i<j \leq n$

$$
t i l=\left\{\left(t_{1}, t_{2}\right),\left(t_{3}, t_{4}\right), \ldots,\left(t_{n-1}, t_{n}\right)\right\} .
$$

Definition 4.3. For a given set of event instances $S$ (where $S=\left\{i n s_{1}, \ldots\right.$,ins $\left.s_{n}\right\}$ and $n \geq 2$ ), the co-occurrence time interval list (denoted as $t i^{c o}$ ) contains time intervals where there exists a spatiotemporal overlap among all the instances in $S$.

In Figure 4, we illustrated three example co-occurrences. To better explain the concept, we will present the co-occurrence time intervals for each of these co-occurrences. 
-In Figure 4(a), the $t i l^{c o}$ for $i n s_{A}$ and $i n s_{B}$ is $\left[t_{2}, t_{4}\right]$ as these two instances overlap between these time intervals.

- Similarly, in Figure 4(b), the $t i l^{c o}$ for $i n s_{C}$ and $i n s_{D}$ is $\left[t_{3}, t_{5}\right]$.

-In Figure 4(c),

$-t i l^{c o}$ for $i n s_{E}$ and $i n s_{F}$ is $\left[t_{4}, t_{5}\right]$.

$-t i l^{c o}$ for $i n s_{E}$ and $i n s_{G}$ is $\left[t_{3}, t_{5}\right]$.

$-t i l^{c o}$ for $i n s_{F}$ and $i n s_{G}$ is $\left[t_{4}, t_{7}\right]$.

$-t i l^{c o}$ for $i n s_{E}$, ins $s_{F}$, and $i n s_{G}$ is $\left[t_{4}, t_{5}\right]$ as between $t_{4}$ and $t_{5}$ all three of $i n s_{E}$, ins $F$, and ins $_{G}$ spatiotemporally overlap. This is essentially the intersection of $t i l^{c o}$ 's of (ins $\left.s_{E}, i n s_{F}\right)$, $\left(\right.$ ins $\left._{E}, i n s_{G}\right)$, and $\left(i n s_{F}, i n s_{G}\right)$.

For the $J^{+}$measure, the amount of geometric calculations (i.e., determining the union and intersection of instances, calculating the areas and volume) is limited to the co-occurrence time intervals. Space requirement is also reduced, because for each co-occurrence the intersection and union geometries are included only if there exists a spatiotemporal overlap among all participating instances. However, the $J^{+}$measure has potential drawbacks regarding the filtering mechanism (i.e., elimination of particular segments of spatiotemporal instances).

For a spatiotemporal co-occurrence which has three or more participating instances, the cooccurrence time interval list only includes the time intervals in which trajectories of all participating instances overlap. Nevertheless, any event of co-occurrence between two instances is a region of interest, and should be considered. These regions can be disregarded when calculating the $J^{+}$. Another problem stemming from the same issue is the antimonotonic property of $J^{+}$. STCOP mining algorithms efficiently employ a downward closure property, and require the significance measures to carry an antimonotonic property.

LEMma 4.4. $J^{+}$measure is not antimonotonic.

Proof. We will present a proof by contradiction. Assume that $J^{+}$measure is antimonotonic. In Figure 5, the locations of three spatiotemporal instances for four different timestamps are demonstrated, as well as the corresponding area values of instances, their intersections, and unions for each timestamp. Let the time difference between each timestamp be $\left(\tau=t_{i}-t_{i-1}\right)$. Then, the $J^{+}$value of any spatiotemporal co-occurrence of two instances (i.e., $J^{+}\left(i n s_{1}, i n s_{2}\right)$, $\left.J^{+}\left(i n s_{1}, i n s_{3}\right), J^{+}\left(i n s_{2}, i n s_{3}\right)\right)$ must be greater than or equal to the $J^{+}$value of any spatiotemporal co-occurrence of three instances (i.e., $\left.J^{+}\left(i n s_{1}, i n s_{2}, i n s_{3}\right)\right) . J^{+}\left(i n s_{1}, i n s_{2}\right)$ is $\frac{\tau(10+80+70)}{\tau(145+90+85)}=0.5$ and $J^{+}\left(i n s_{1}, i n s_{2}, i n s_{3}\right)$ is $\frac{\tau(80)}{\tau(120)} \tilde{=} 0.66$. This contradicts the earlier assumption of the $J^{+}$measure being antimonotonic as the value of the measure for this particular example increased as the cardinality of the co-occurrence increased. Therefore, the $J^{+}$is not an antimonotonic measure.

\section{$5 \quad J^{*}$ MEASURE}

A problem with the $J^{+}$is that it does not consider the spatiotemporal co-occurrences appearing in the subsets of participating instances, but only considers the intervals that all participating instances spatiotemporally overlap. For the example shown in Figure 5, the $J$ measure would incorporate all the geometries (from $t_{1}$ to $t_{4}$ ) for all instances when calculating the union volumes (whether there exists a co-occurrence or not). On the other end of spectrum, the $J^{+}$measure only calculates the union volume for the co-occurrence among all the participating instances, but does not reflect any information about the co-occurrences among the subsets of the participating instances. Accordingly, to eradicate the problems regarding the $J$ measure and avoid the neglect of the subset co-occurrences, we introduce the concept of cross co-occurrences, which provides a foundation for the antimonotonic $J^{*}$ measure. 


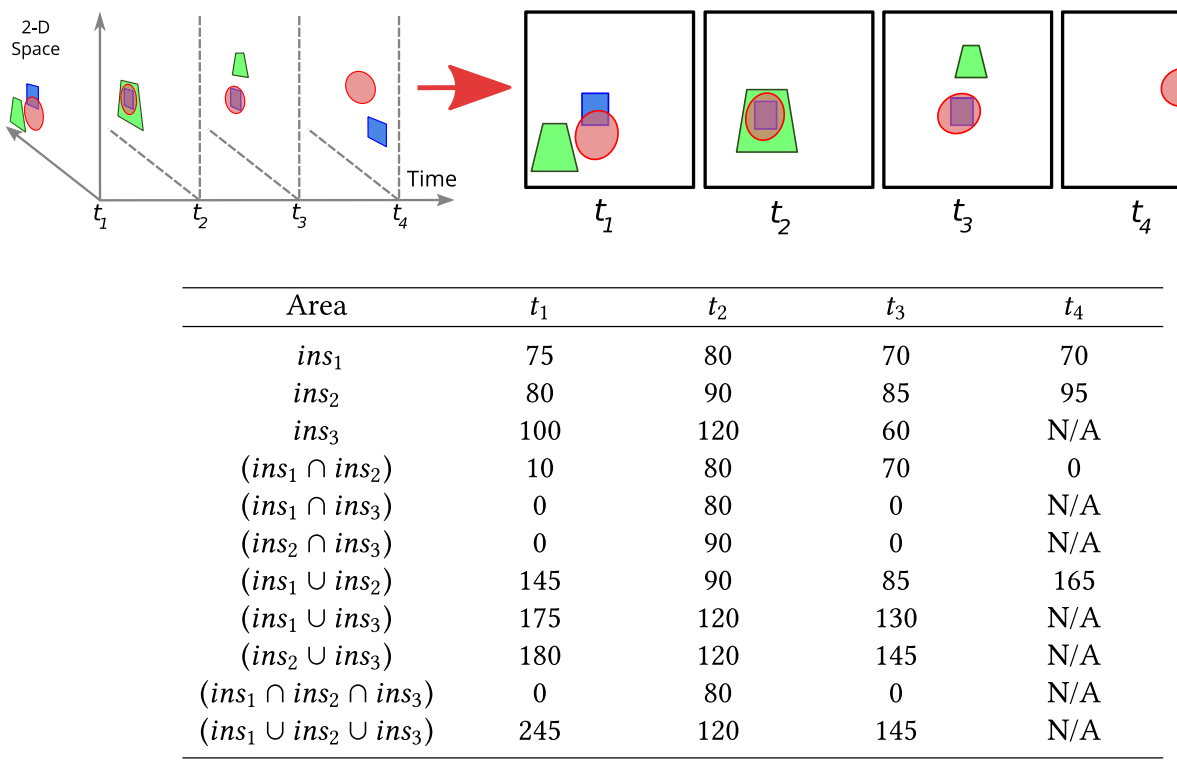

Fig. 5. An example co-occurrence of three spatiotemporal instances with the area values at particular timestamps.

Definition 5.1. A set of cross co-occurrences $(x c o)$ in a spatiotemporal co-occurrence is the spatiotemporal overlap relationships, which occurred among the 2-subsets of participating instances.

Definition 5.2. For a given set of event instances $S$ (where $S=\left\{i n s_{1}, \ldots, i n s_{n}\right\}$ and $n \geq 2$ ), the cross co-occurrence time interval list (denoted as $t i^{x c o}$ ) contains time intervals where there exists a spatiotemporal overlap among at least two instances in $S$. Alternatively, let $S u b S$ be a 2 -subset of $S$ such that $S u b S=\left\{\right.$ ins $_{i_{1}}$, ,ins $\left._{i_{2}}\right\}$, where $1 \leq i_{1}<i_{2} \leq n$. Then, the cross co-occurrence time interval list for $S$ is the temporal union of the co-occurrence time interval lists of each 2-subset of $P$.

$$
t i l^{x c o}=\bigcup_{S u b S \subseteq S} t i l^{c o}(S u b S),
$$

where $t i l^{c o}(S u b S)$ denotes the co-occurrence time interval list of instances in SubS.

Let $J^{*}$ be a significance measure for spatiotemporal co-occurrences. We define the $J^{*}$ measure using the interval volume function (in Definition 4.1) and the cross co-occurrence time interval list (in Definition 5.2) as follows:

$$
J^{*}\left(i n s_{1}, \ldots, \text { ins }_{n}\right)=\frac{V_{t i l^{\text {xco }}}\left(i n s_{1} \cap \ldots \cap i n s_{n}\right)}{V_{t i l^{\text {xco }}}\left(i n s_{1} \cup \ldots \cup i n s_{n}\right)} .
$$

Although the $J, J^{+}$, and $J^{*}$ measures might seem similar in notation, they are considerably different because of the variations stemming from the interpretations of co-occurrence and cross co-occurrence time interval lists in their respective volume functions. For clarification, we present an example following the instances shown in Figure 5. The $J^{*}$ and $J^{+}$values are equal for size-2 co-occurrences because cross co-occurrence time intervals are the same with co-occurrence time intervals. However, the $J^{*}$ for size- 3 spatiotemporal co-occurrence of $i n s_{1}$, ins $s_{2}$, and $i n s_{3}$ is different from $J^{+}$and thus, calculated as follows. Firstly, the co-occurrence time intervals $\left(t i l^{c o}\right)$ for each 2 -subset are determined. 
- For ins $s_{1}$ and $i n s_{2}-$ til $^{c o}\left(\right.$ ins $\left._{1}, i n s_{2}\right)=\left[t_{1}, t_{4}\right)$.

- For $i n s_{1}$ and $i n s_{3}-t i l^{c o}\left(\right.$ ins $_{1}$, ins $\left._{3}\right)=\left[t_{2}, t_{3}\right)$.

- For $i n s_{2}$ and $i n s_{3}-t i l^{c o}\left(i n s_{2}, i n s_{3}\right)=\left[t_{2}, t_{3}\right)$.

Then, the cross co-occurrence time interval list $t \operatorname{li}^{x c o}\left(\mathrm{ins}_{1}, i n s_{2}, i n s_{3}\right)$ is

$$
t i l^{x c o}\left(i n s_{1}, i n s_{2}, i n s_{3}\right)=t i l^{c o}\left(i n s_{1}, i n s_{2}\right) \cup t i l^{c o}\left(i n s_{1}, i n s_{3}\right) \cup t i l^{c o}\left(i n s_{2}, i n s_{3}\right)=\left[t_{1}, t_{4}\right) .
$$

For the cross co-occurrence time interval, $\left[t_{1}, t_{4}\right)$,

- The intersection volume is $V_{\left\{\left[t_{1}, t_{4}\right)\right\}}\left(\right.$ ins $_{1} \cap$ ins $\left._{2} \cap i n s_{3}\right)=\tau(0+80+0)$.

- The union volume is $V_{\left\{\left[t_{1}, t_{4}\right)\right\}}\left(\right.$ ins $\left._{1} \cup i n s_{2} \cup i n s_{3}\right)=\tau(245+120+145)$.

-Then, the $J^{*}$ value is $J^{*}\left(i n s_{1}, i n s_{2}, i n s_{3}\right)=\frac{V_{\left\{\left[t_{1}, t_{4}\right)\right\}}\left(i n s_{1} \cap i n s_{2} \cap i n s_{3}\right)}{V_{\left\{\left[t_{1}, t_{4}\right)\right\}}\left(i n s_{1} \cup i n s_{2} \cup i n s_{3}\right)}=\frac{80 \tau}{510 \tau} \tilde{=} 0.16$.

Earlier, we calculated the $J^{+}$value for the same instances as 0.66 . The intersection volume remains unchanged; however, as the co-occurrence time interval is $\left[t_{2}, t_{3}\right)$, the union volume for $J^{+}$ is calculated as $120 \tau$, which is different from $510 \tau$ for $J^{*}$.

The $J^{*}$ measure, unlike the $J$ measure, does not particularly favor the instances, which carry similar volume characteristics. It acknowledges the spatiotemporal co-occurrence of all participating instances as the main event of interest, while also considering the cross co-occurrences appearing among the subsets of participating instances. Regardless of the unbalanced characteristics instances may have, the regions of interest for $J^{*}$ measure are only limited to co-occurrence and cross co-occurrences. Therefore, the $J^{*}$ can be considered less biased when handling the coverage anomalies and bias created in the co-occurrences by small or large volume instances.

Another important aspect of the problem is the storage requirements and computational complexity. For all measures derived from Jaccard $\left(J, J^{+}, J^{*}\right)$, the numerator in the ratio is the volume of the spatiotemporal intersection of all instances. However, the denominator, which calculates union volume, changes drastically. In particular, for long-lasting events, storage of the union volumes may create huge storage overhead. From a practical point of view, storing only cross cooccurrences can greatly reduce storage requirements. The geometric calculations for determining unions and intersections are typically very expensive operations. Theoretically, the upper bound of geometric calculations for the $J, J^{+}$, and $J^{*}$ measures are the same (consider the case where all instances overlap at all time intervals).

\subsection{Algorithms for $\mathrm{J}^{*}$ Calculation}

We introduce two algorithms for calculating the $J^{*}$ measure. Our first algorithm (shown in Algorithm 1) is designed for calculating $J^{*}$ measure for two event instances. The second one is the generalized algorithm (shown in Algorithm 2). It calculates the $J^{*}$ measure for two or more event instances. In both of our algorithms, we consider instances are modeled as a list of timestampgeometry pairs [3]. The list of auxiliary functions used in $J^{*}$ calculation algorithms are listed, and their descriptions are demonstrated in Table 2.

In Algorithm 1, two spatiotemporal instances are given as input. Initially, intervals where the lifespans of two instances overlap are discovered (i.e., coexistence time interval list, $\mathrm{til}^{\mathrm{ce}}$ ). Then, for each interval in $t i l^{c e}$, we find the intersection area. If the geometries intersect (iArea $\left.>0\right)$ at a given time interval, we calculate the union area. Later, we increase the intersection and union volumes using the intersection and union areas. If there is no spatiotemporal intersection between two instances, the algorithm returns 0 ; else, it returns the ratio between intersection and union volumes. 
Table 2. Auxiliary Functions Used in $J^{*}$ Calculations

\begin{tabular}{|c|c|}
\hline Function & Description \\
\hline \multirow{3}{*}{ FindCrossCooccurrence(instances) } & $\begin{array}{l}\text { The function returns a coexistence time interval list } \\
\left(t i l^{c e}\right) \text { that contains intervals where the lifespans of } \\
\text { two given instances }\left(i n s_{1}, i n s_{2}\right) \text { temporally overlap. }\end{array}$ \\
\hline & $\begin{array}{l}\text { The function returns the cross co-occurrence time } \\
\text { interval list }\left(t i l^{x c o} \text {; see Definition 5.2) of a set of }\right. \\
\text { instances. }\end{array}$ \\
\hline & $\begin{array}{l}\text { This function is applied to a spatiotemporal instance } \\
\text { (ins). It returns the region geometry of the instance } \\
\text { at the given time interval }(i v l) \text {. }\end{array}$ \\
\hline$\langle$ ins $\rangle$.GetTimeIntervals() & $\begin{array}{l}\text { The function returns the set of uniformly sampled } \\
\text { time intervals of the instance (ins). }\end{array}$ \\
\hline$\langle$ Collection $\rangle$.Insert(item) & The function inserts an item to a collection. \\
\hline Intersection (geometries) & $\begin{array}{l}\text { The function returns the spatial intersection } \\
\text { geometry of a given collection of geometries. }\end{array}$ \\
\hline Union(geometries) & $\begin{array}{l}\text { The function returns the spatial union geometry of a } \\
\text { given collection of geometries. }\end{array}$ \\
\hline Intersects $\left(g_{1}, g_{2}\right)$ & $\begin{array}{l}\text { The function returns true if two given geometries } \\
\left(g_{1}, g_{2}\right) \text { spatially intersect; otherwise, returns false. }\end{array}$ \\
\hline Area (geometry) & The function returns the area of the given geometry. \\
\hline
\end{tabular}

In Algorithm 2, using the FindCrossCooccurrence procedure an initial cross co-occurrence time interval list $\left(t i l^{x c o}\right)$ is found. The procedure iterates over each 2-subsets of given instances. Firstly, for every possible pair, the procedure finds the coexistence time intervals, and later discovers the co-occurrence time intervals by checking the spatial overlap (see Definition 4.3, $t_{i} l^{x c o}$ ). The union of all co-occurrence time intervals gives the cross co-occurrence time intervals. After discovering $t i l^{x c o}$, the algorithm iterates over the intervals in $t i l^{x c o}$ for volume calculations. In each iteration, intersection and union areas are found, and intersection and union volumes are increased accordingly. If there is no spatiotemporal intersection among the instances, the algorithm returns 0 ; else, it returns the ratio between intersection and union volumes.

In a nutshell, our algorithms initially determine the temporal co-existence, and later check for the spatial overlap between individual geometries. Therefore, we eliminate the computationally expensive spatial intersection and union operation when they are not necessary. Both of our algorithms effectively calculate the intersection and union volumes at cross co-occurrence time intervals. In Algorithm 1, for two instances, co-occurrence and cross co-occurrence time intervals are the same, and the volumes are simultaneously discovered. In Algorithm 2, the cross co-occurrence time intervals are discovered in advance. Later, the intersection and union volumes are calculated.

\subsection{Key Properties of $\mathrm{J}^{*}$}

In this section, two key properties of $J^{*}$, which are related to spatiotemporal frequent pattern mining, will be discussed. The first one is the antimonotonic property, which is vital for efficiency and correctness of STCOP mining. The second one is the containment property, which shows the relation between the $J, J^{+}$, and $J^{*}$ measures.

5.2.1 Antimonotonic Property. Downward closure property (i.e., antimonotonicity) is the fundamental aspect of many objective measures used in frequent pattern mining. Similar to the 


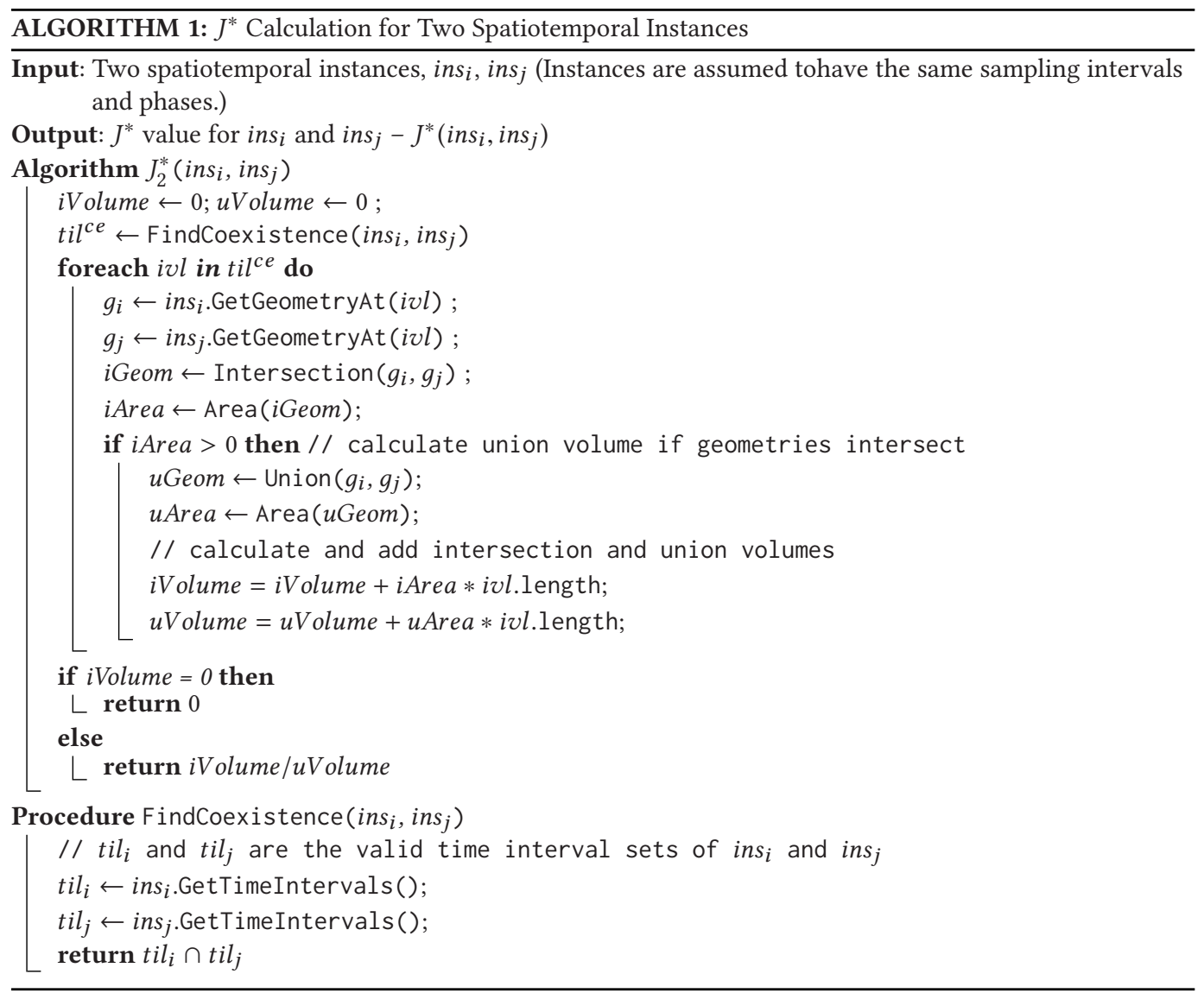

seminal frequent pattern mining approaches [1,23], in STCOP mining (which are based on the Apriori algorithm [1]) the antimonotonicity plays a significant role for efficiently and correctly mining the co-occurrences.

\section{LEMMA 5.3. $J^{*}$ is an antimonotonic measure.}

Proof. Let $S$ be the set of participating instances of a spatiotemporal co-occurrence $(S=$ $\left.\left\{i n s_{1}, \ldots, i n s_{n}\right\}\right)$. Let $i n s_{n+1}$ be another instance that forms a co-occurrence with all the instances in $S$. Then $S^{\prime}=\left\{i n s_{1}, \ldots\right.$, ins $\left.n, i n s_{n+1}\right\}$ and $S \subset S^{\prime}$. Then, $J^{*}(S) \geq J^{*}\left(S^{\prime}\right)$, because

(1) $V_{t i l^{x c o}}\left(i n s_{1} \cap \ldots \cap i n s_{n} \cap i n s_{n+1}\right) \leq V_{t i l^{x c o}}\left(i n s_{1} \cap \ldots \cap i n s_{n}\right)$. The intersection volume can only decrease or stay the same with the addition of a new instance to the participating instance set.

(2) $V_{t i l^{x c o}}\left(i n s_{1} \cup \ldots \cup i n s_{n} \cup i n s_{n+1}\right) \geq V_{t i l^{x c o}}\left(i n s_{1} \cup \ldots \cup i n s_{n}\right)$. The union volume can only increase or stay the same with the addition of a new instance to the participating instance set. Note that the cross co-occurrence time interval list of $S^{\prime}$ includes at least the cross co-occurrence time interval list of $S$, and it can potentially include cross co-occurrences between $i n s_{n}$ and the instances in $S\left(S . t i l^{x c o} \subseteq S^{\prime} . t i l^{x c o}\right)$. Therefore, the union volume for $S^{\prime}$ is greater than or equal to the union volume of $S$. 


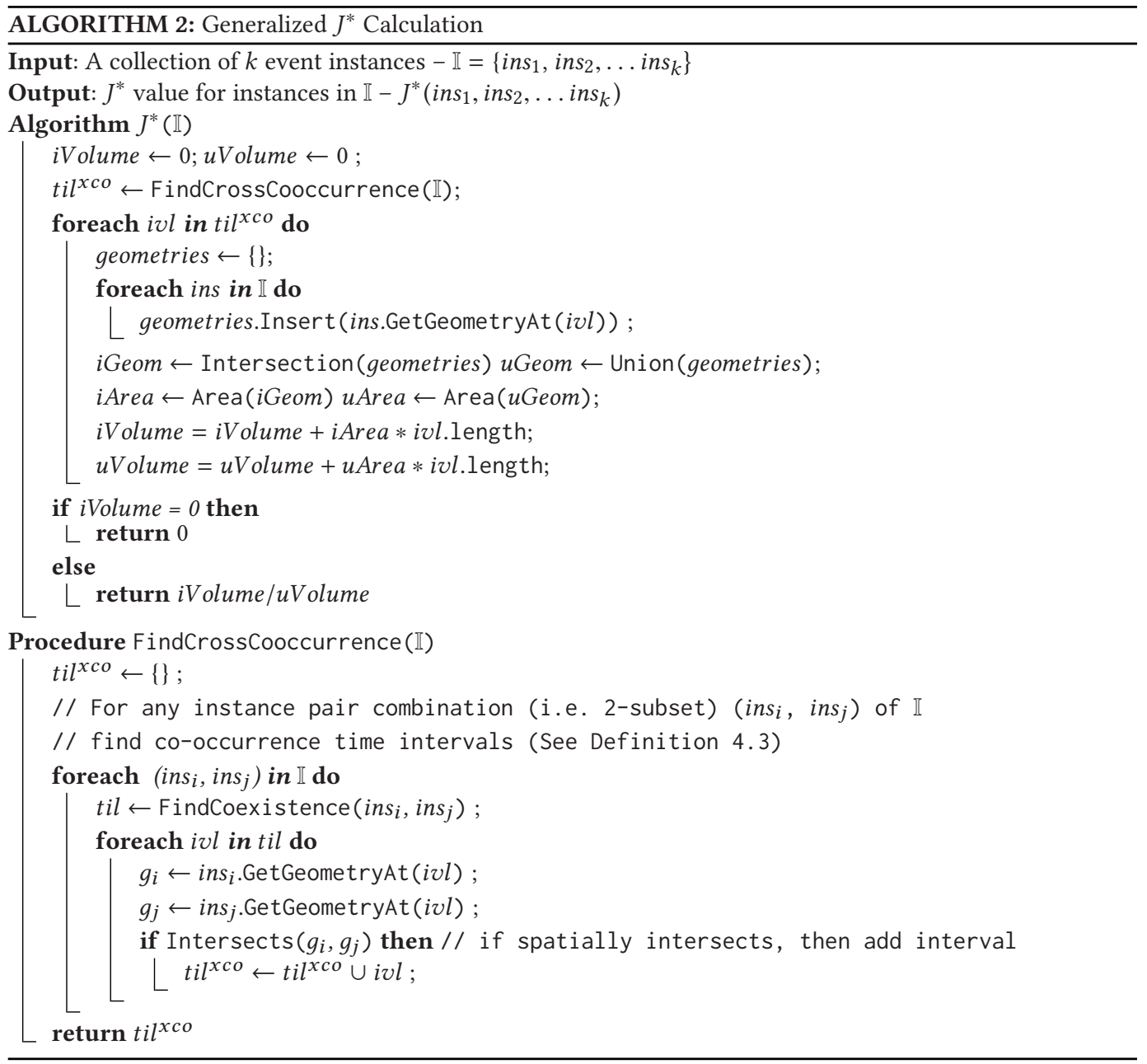

The $J^{*}$ value for a co-occurrence decreases or stays the same with the addition of a new spatiotemporal instance, as the intersection volume can only decrease or stay the same and the union volume can only increase or stay the same. Hence, $J^{*}$ is an antimonotonic measure.

5.2.2 Containment Property. The interestingness of discovered patterns is an important aspect of the data mining research. The concept of interestingness for a pattern includes characteristics such as conciseness, generality, surprisingness, and novelty [21]. The $J$ measure can be considered to provide generality as a large fraction of discovered knowledge matches the well-known patterns. However, its ability to discover unexpected or obscure co-occurrences is limited due to the shortcomings we have addressed in Section 3. With the $J^{+}$and $J^{*}$ measures, we aim to achieve novelty and possible surprisingness (meaning not known before, or contradicting the existing knowledge), while preserving generality.

The containment relationship between the $J, J^{*}$, and $J^{+}$measures dictates that for any spatiotemporal co-occurrence, the value of the $J$ is always less than or equal to the value of $J^{*}$ and the value of the $J^{*}$ is always less than or equal to the value of $J^{+}$. The containment relationship 
is important, as it helps to maintain the desired generality related characteristics of $J$ measure. The property can be described as follows: Given a particular co-occurrence coefficient threshold, if a spatiotemporal co-occurrence is assessed as significant based on $J$ value, it is also significant based on $J^{*}$ value. Similarly, if a spatiotemporal co-occurrence is assessed as significant based on $J^{*}$ value, it is also significant based on $J^{+}$value.

\section{Lemma 5.4. $J^{+}$measure contains $J^{*}$ measure, and $J^{*}$ measure contains $J$ measure.}

Proof. Let $S$ be the set of participating instances of a spatiotemporal co-occurrence $(S=$ $\left.\left\{i n s_{1}, \ldots, i n s_{n}\right\}\right)$. The $J$ value for a particular co-occurrence can never be greater than the $J^{*}$ value, and the $J^{*}$ value can never be greater than the $J^{+}$value $\left(J^{+}(S) \geq J^{*}(S) \geq J(S)\right)$ :

(1) $V_{t i l}$ co $\left(i n s_{1} \cap \ldots \cap i n s_{n}\right)=V_{t i l}$ xco $\left(i n s_{1} \cap \ldots \cap i n s_{n}\right)=V\left(i n s_{1} \cap \ldots \cap i n s_{n}\right)$. Intersection volumes calculated for both measures are equal, as $t i l^{c o}$ and $t i l^{x c o}$ include the interval of the co-occurrence of all the participating instances.

(2) $V_{t i l^{x c o}}\left(i n s_{1} \cup \ldots \cup i n s_{n}\right) \leq V\left(i n s_{1} \cup \ldots \cup i n s_{n}\right)$. Union volume calculated for $J^{*}$ is less than or equal to the union volume calculated for the $J$ measure, since the $V_{t i l}$ xco function only calculates the union volume for the time intervals specified in $t i^{x c o}$, and the intervals in $t i l^{x c o}$ are a subset of the intervals specified by the lifespans of all participating instances.

(3) Similarly, $V_{t i l}$ co $\left(i n s_{1} \cup \ldots \cup i n s_{n}\right) \leq V_{t i l c o}\left(i n s_{1} \cup \ldots \cup i n s_{n}\right)$. The union volume calculated for $J^{+}$is less than or equal to the union volume calculated for the $J^{*}$ measure, since the $t i l^{x c o}$ is a superset of $t i l^{c o}$.

For any spatiotemporal co-occurrence, the $J^{+} \geq J^{*} \geq J$ because intersection volumes are the same for all the measures and the union volumes have the relationship $V_{t i l^{c o}} \leq V_{t i l^{x c o}} \leq V$. Hence, $J$ is contained by $J^{*}$ and $J^{*}$ is contained by $J^{+}$.

\section{EXPERIMENTAL EVALUATION}

In this section, we will evaluate the relevancy and efficiency of the $J, J^{+}, J^{*}, O M I N$, and OMAX measures. The $J, O M A X$, and OMIN measures are previously used for STCOP mining in [10, 46, 48]. In the Online Appendix of this article (Part B), we show the algorithms for the OMIN, OMAX, $J^{+}$, and $J$ calculations.

We have conducted our experiments for real-life solar event datasets and four artificial datasets with varying spatiotemporal characteristics. The artificial datasets are generated using the random dataset generator [9]. The solar event data is partitioned into four datasets, each corresponding to 3-month periods (quarters) in 2012. For relevancy analysis, we used the solar event datasets. For efficiency analysis, we used both artificial and solar event datasets. In our experiments, we have enumerated all the size- 2 and size- 3 spatiotemporal co-occurrences among the instances of all different event types. We reported the $J, J^{+}, J^{*}, O M I N$, and $O M A X$ values and running times for each spatiotemporal co-occurrence.

\subsection{Experimental Settings}

Our real-life solar event datasets are obtained from Heliophysics Event Knowledgebase [29]. The individual recordings of the solar events are tracked and interpolated using the algorithms in [32] and [20]. The solar event datasets include the instances of seven solar event types that are (1) Active Regions (AR), (2) Coronal Holes (CH), (3) Emerging Flux (EF), (4) Filaments (FI), (5) Flares (FL), (6) Sigmoids (SG), and (7) Sunspots (SS). The artificial datasets have two event types. The details of these datasets can be found in Table 3. The significance measures are implemented in the Java programming language. We used Algorithm 1 and Algorithm 2 for $J^{*}$ calculations. The 
Table 3. Datasets Used in the Experiments

\begin{tabular}{|c|c|c|c|c|c|c|}
\hline \multirow{5}{*}{$\begin{array}{c}\text { Solar } \\
\text { Event } \\
\text { Datasets }\end{array}$} & \multicolumn{2}{|c|}{ Dataset } & Start Date & End Date \# & \# of Polygons \# & \# of Instances \\
\hline & \multirow{2}{*}{$\begin{array}{l}\text { Quarter } 1 \text { - } 2012 \\
\text { Quarter } 2 \text { - } 2012\end{array}$} & $\begin{array}{ll}012 & \text { Q1 }\end{array}$ & $01 / 01 / 2012$ & $03 / 31 / 2012$ & 439,512 & 6,498 \\
\hline & & 012 Q2 & $04 / 01 / 2012$ & $06 / 30 / 2012$ & 537,078 & 7,911 \\
\hline & Quarter 3 - 2012 & $012 \quad \mathrm{Q} 3$ & 07/01/2012 & 09/30/2012 & 570,875 & 8,527 \\
\hline & \multicolumn{2}{|c|}{ Quarter 4 - 2012} & 10/01/2012 & $12 / 31 / 2012$ & 503,001 & 6,854 \\
\hline \multirow{5}{*}{$\begin{array}{l}\text { Artificial } \\
\text { Datasets }\end{array}$} & Dataset & Tag & $\begin{array}{l}\text { \# of Vertices } \\
\text { per Polygon }\end{array}$ & $\begin{array}{c}\text { \# of tpgs } \\
\text { per Instance }\end{array}$ & e \# of Polygons & $s$ \# of Instances \\
\hline & $\begin{array}{r}\text { Low Vertex } \\
\text { Short Lifespan }\end{array}$ & LVSL & 20 & 20 & 20,000 & 1,000 \\
\hline & $\begin{array}{r}\text { Low Vertex } \\
\text { Long Lifespan }\end{array}$ & LVLL & 20 & 100 & 100,000 & 1,000 \\
\hline & $\begin{array}{l}\text { High Vertex } \\
\text { Short Lifespan }\end{array}$ & HVSL & 100 & 20 & 20,000 & 1,000 \\
\hline & $\begin{array}{l}\text { High Vertex } \\
\text { Long Lifespan }\end{array}$ & HVLL & 100 & 100 & 100,000 & 1,000 \\
\hline
\end{tabular}

algorithms for the $J, J^{+}, O M A X$, and OMIN measures are shown in the Online Appendix, Part B. For reproducibility of the experiments, the datasets, result sheets, and our source code can be found on our project website [2].

\subsection{Relevancy Analysis}

In this part of our discussion, we will discuss the relevancy of our new measures, $J^{+}$and $J^{*}$. In Section 3, we have outlined the possible anomalies created due to unfair assessments of the $J$ measure. We will analyze each of the mentioned anomalies for solar event data, compare the measures with $O M A X$ and $O M I N$ measures, and how the $J^{*}$ measure addresses these particular problems.

In Figure 6, we demonstrate boxplots showing the value distributions of $J, J^{+}, J^{*}, O M I N$, and $O M A X$ measures for size- 2 and size- 3 co-occurrences in the $\mathrm{Q} 1$ dataset. The results from other solar event datasets can be found in the Online Appendix of this article (Part C). The co-occurrences are grouped by the event types of participating instances. In size- 2 co-occurrences, the $J^{+}$and $J^{*}$ values are merged as we have observed that they were the same. Additionally, the medians and means of each distribution are represented as red lines and magenta dots.

From Figure 6, by analyzing the mean and median points and the confidence intervals of the distributions, we can see the following:

-OMAX and $J$ measures have similar distributions.

$-J^{*}$ values are generally higher than $J$ and OMAX values, and lower than $J^{+}$and OMIN measures.

- The OMIN and $J^{+}$measures have the highest values.

- The confidence interval for the $J^{+}$measure is usually smaller than the others.

- When compared to the $J^{*}$ or $J^{+}$values, the $J$ and $O M A X$ values have higher chances of being an outlier within their own data series. This situation is more noticeable particularly for the co-occurrences that involve the event instances of Flare (FL).

$-O M I N$ measure creates more outliers when spatiotemporal characteristics of the event types are similar. 

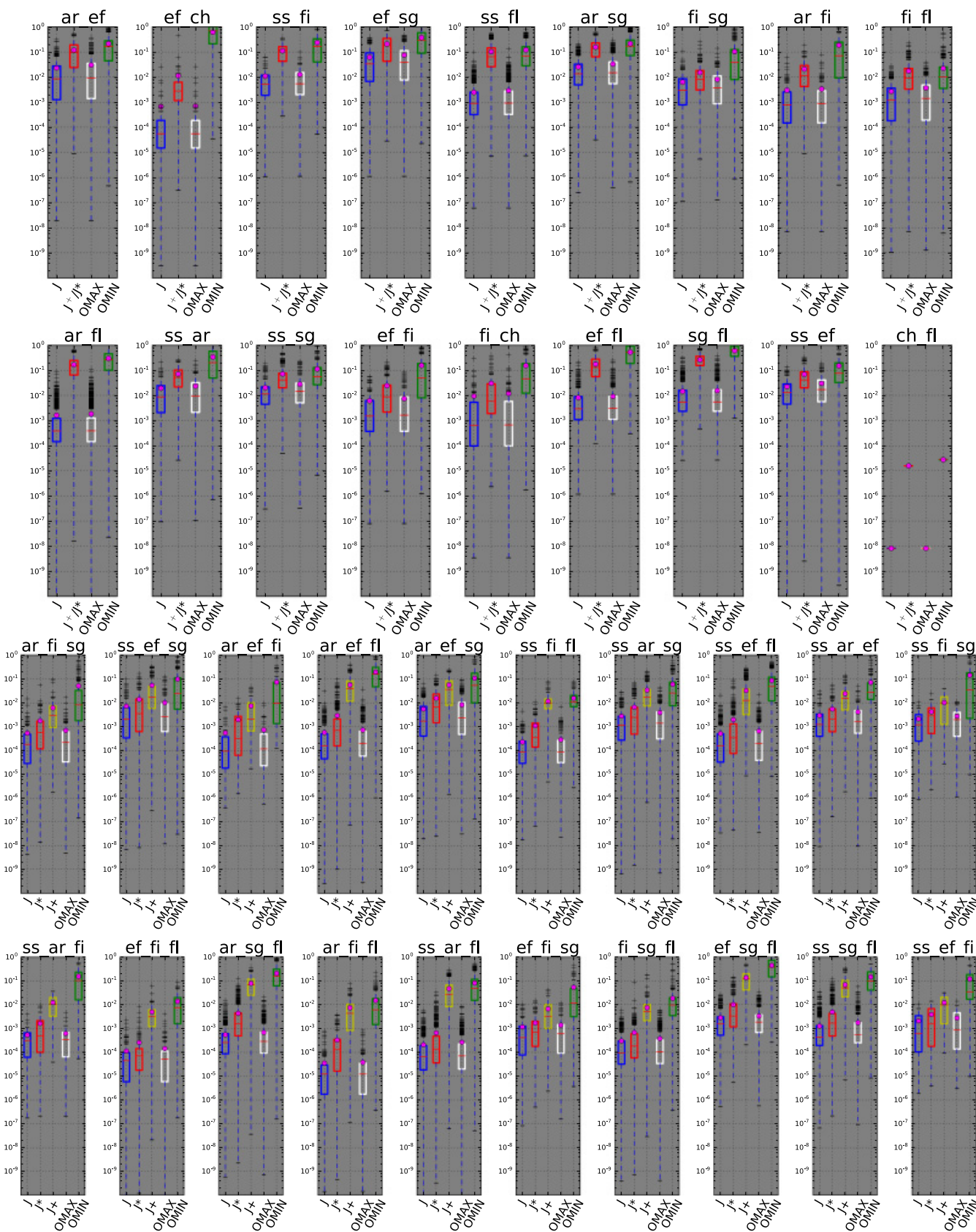

Fig. 6. The boxplots showing the distribution of $J, J^{+}, J^{*}, O M I N$, and $O M A X$ values (in logarithmic scale) for size-2 and size-3 spatiotemporal co-occurrences in the Q1 dataset. Each subfigure shows co-occurrences between different event types. For size- 2 co-occurrences $J^{+}$and $J^{*}$ are joined since they output the same value. $J, J^{+}, J^{*}, O M I N$, and $O M A X$ values are represented with blue, yellow, red, white, and green boxes, respectively. 


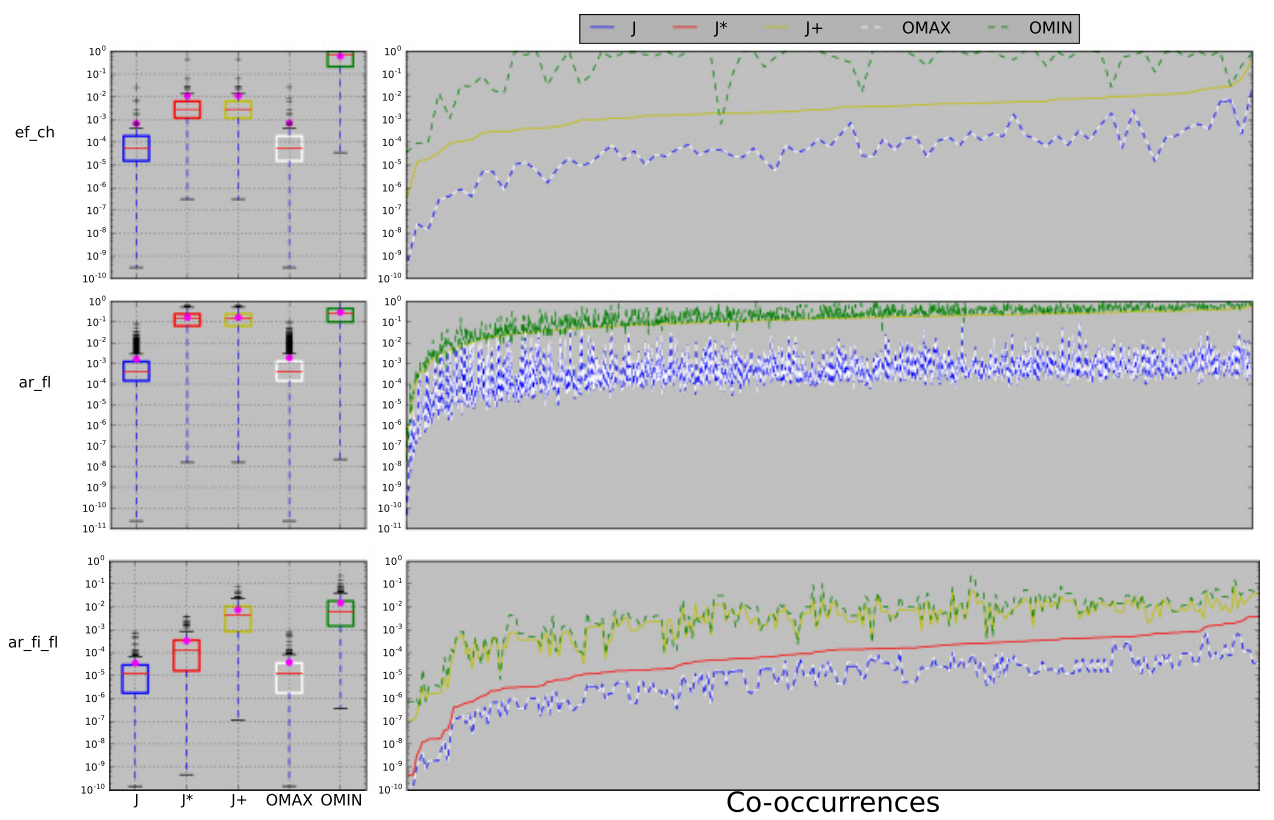

Fig. 7. The value distributions of significance measures for EF-CH, AR-FL, and AR-FI-FL co-occurrences. Boxplots are demonstrated on the left; value comparison plots for individual co-occurrences are displayed on the right (sorted on $J^{*}$ ).

- The greater variations among the value distributions are from the co-occurrences between the instances of event types with very different characteristics such as EF-CH, SG-FL, or AR-SG-FL.

- There are no theoretical containment relationships between our new measures $\left(J^{+}\right.$or $\left.J^{*}\right)$ and overlap measures $(O M A X$ and $O M I N)$. However, for both mean and median values, we see the following trend: $J<O M A X<J^{*} \leq J^{+}<O M I N$.

In Figure 6, we can observe that the co-occurrences of EF-SG, SS-AR, AR-SG, SS-SG, and SG-FL have relatively higher $J$ and $O M A X$ values. On the other hand, the co-occurrences between AR-EF, EF-SG, AR-SG, AR-FL, EF-FL, and SG-FL have higher $J^{*}$ (or $J^{+}$) and $O M I N$ values. We can suggest that the significance assessments with $J^{+}, J^{*}$, or OMIN measures increase the likelihood of a small volume instance (such as EF or FL) to be involved in a significant co-occurrence given a particular cce threshold value. Next, we will analyze the anomalies that we have mentioned in Section 3, and how the $J^{*}$ addresses these anomalies. Additionally, we will analyze the top-10 most significant co-occurrences discovered in each of the five measures.

6.2.1 Coverage Anomaly. In Figure 3, we have demonstrated the spatiotemporal characteristics of different event types. Instances of event types such as $\mathrm{AR}$ and $\mathrm{CH}$ have relatively higher volumes. On the other hand, instances of FL or EF event types have smaller volumes. Additionally, instances of FI and SS event types have longer lifespans. The coverage anomaly refers to the unfair significance assessments of co-occurrences between very large and very small volume instances.

Figure 7 exhibits typical coverage anomaly problems between the large volume AR and $\mathrm{CH}$ instances and small volume EF and FL instances. Flares can occur anywhere on the Sun's surface, from active regions to the boundaries of the magnetic network of the quiet Sun [34]. 

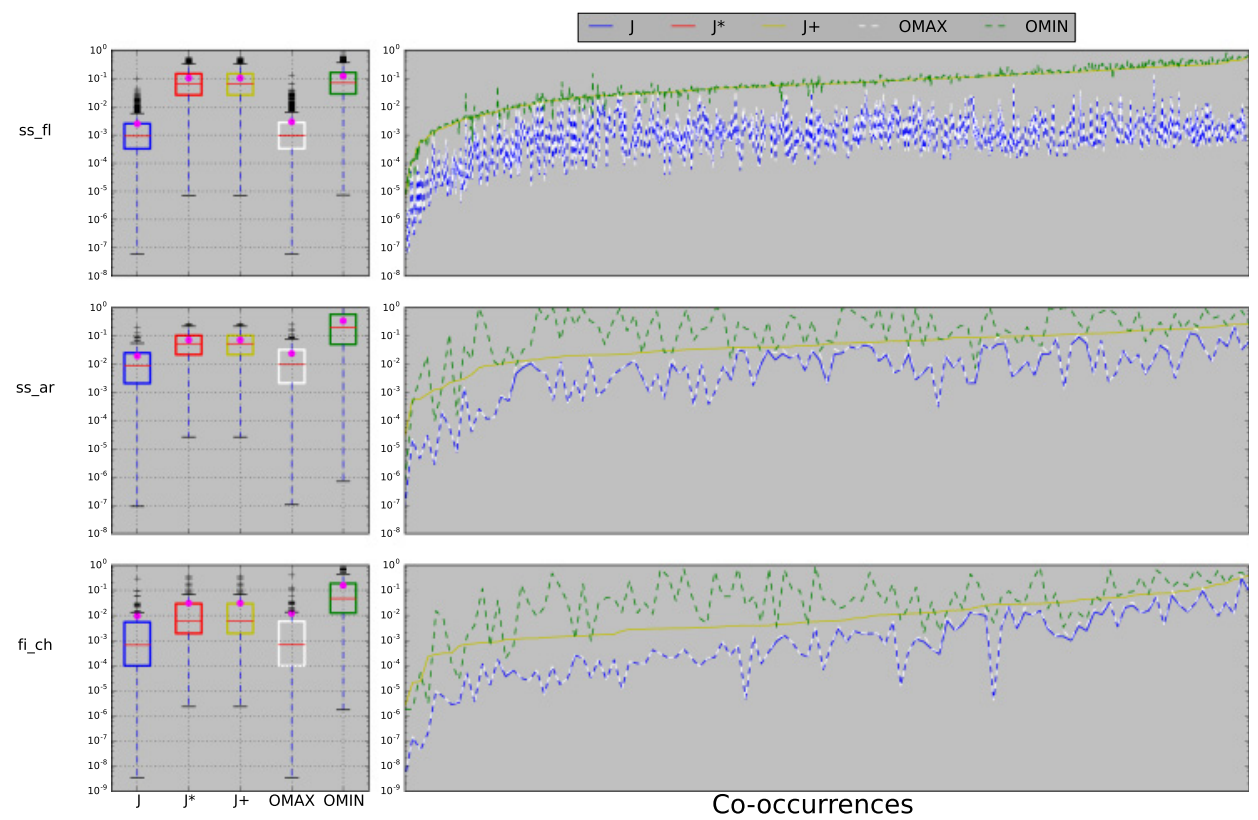

Fig. 8. The distributions of significance measures for SS-FL, SS-AR, and FI-CH co-occurrences. Boxplots are demonstrated on the left; value comparison plots for individual co-occurrences are displayed on the right (sorted on $J^{*}$ ).

However, large area flares have preferred locations. They occur inside the large active regions showing a complex geometry of the 3D magnetic field [50]. Similarly, flux tubes (i.e., emerging flux) are also observed to be emerging into coronal holes [43]. The individual OMAX and $J$ values for the above-mentioned co-occurrences in Figure 7 are very similar. We can suggest that small volume $\mathrm{EF}$ and FL instances are mostly covered, because maximum volume (denominator in OMAX) and the union volume (denominator in $J$ ) of co-occurring instances are very similar.

It is apparent that the large volumes of $\mathrm{AR}$ and $\mathrm{CH}$ instances unfairly decrease the $J$ and $O M A X$ values. The mean $J$ value for AR-FL is two orders of magnitude smaller than the $J^{*}$ value. Likewise, the mean $J$ values for EF-CH and AR-FI-FL are more than one order of magnitude smaller than the $J^{*}$ value.

6.2.2 Favoring the Similar. In Figure 8, we demonstrate three different types of size-2 cooccurrences. In Figure 8, the boxplots for measures and individual value comparisons for SS-FL, $\mathrm{SS}-\mathrm{AR}$, and FI-CH co-occurrences are shown.

The sunspot (SS) instances appear inside the active regions (AR), and a flare (FL) is essentially an intense burst of radiation coming from the release of magnetic energy associated with sunspots [40]. Sunspots can last as long as 2 months, while the lifespan of the flares is between mere minutes to several hours. Therefore, AR and SS instances both have relatively longer lifespans, and their volumes are larger, while FL instances have very short lifespans and small volumes. On the other hand, $\mathrm{CH}$ instances have large areas, longer lifespans, and large volumes, and FI instances have medium areas, but longer lifespans. To remind the readers, the $J$ and $O M A X$ values of cooccurrences between instances with similar spatiotemporal characteristics tend to be higher, while the co-occurrences of instances having highly different spatiotemporal characteristics are more likely to have lower $J$ values. 


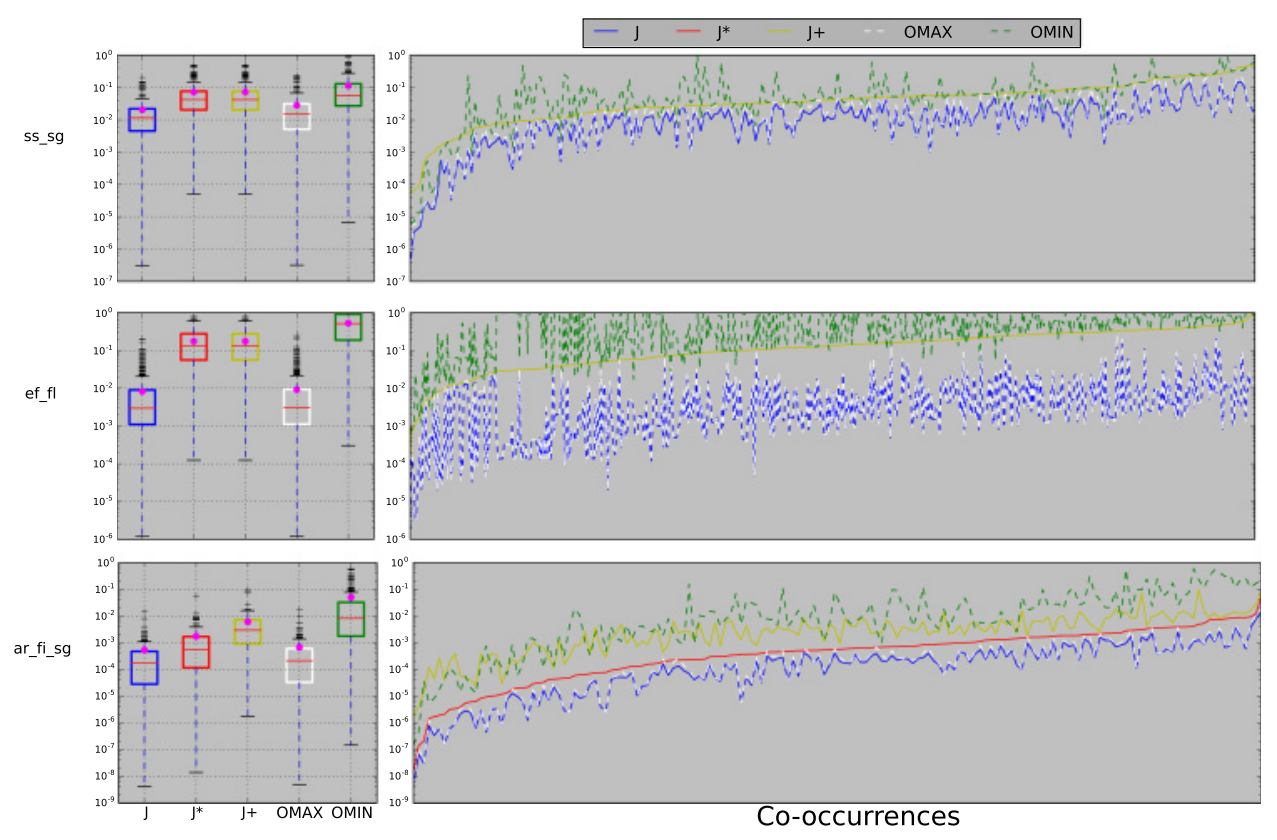

Fig. 9. The value distributions of significance measures for SS-SG, EF-FL, and AR-FI-SG co-occurrences. Boxplots are demonstrated on the left; value comparison plots for individual co-occurrences are displayed on the right (sorted on $J^{*}$ ).

From Figure 8, we can observe that for event types with similar spatiotemporal characteristics (in SS-AR or FI-CH) the $J$ and $O M A X$ values are not significantly increased with $J^{*}$ or $J^{+}$. However, the contrasting spatiotemporal characteristics carried by SS and FL instances drastically affect the $J$ values. A similar situation can be observed for AR and FL instances from Figure 7 . The $J^{*}$ significantly increases the co-occurrence coefficient values for instances with contrasting spatiotemporal characteristics such as AR-FL or SS-FL. Therefore, it can be used for alleviating the favoring the similar problem caused by the $J$ measure.

6.2.3 Large Volume Bias. In Figure 8, we demonstrated the boxplots and value comparisons of $J$ and $J^{*}$ for SS-FL, SS-AR, and FI-CH co-occurrences. In Figure 9, we show the boxplots and value comparisons for SS-SG, EF-FL, and AR-FI-SG co-occurrences. AR, SS, and SG instances have relatively larger volumes, while FI instances have moderate, and EF and FL instances have relatively smaller volumes. SS and FI instances have long lifespans, while SG and AR have larger areas. The large volume bias is created because instances with larger volumes are more likely to have higher $J$ values in a fixed spatial and temporal framework.

From Figures 8 and 9, we can observe that the $J$ values for co-occurrences between instances with large volumes are higher. For both SS-SG or AR-FI-SG co-occurrences, $J^{*}$ does not significantly affect the co-occurrence coefficient. See the $J^{*}$ measure's mean and medians for SS-SG and AR-FI-SG. Contrarily, for co-occurrences involving smaller volume instances, the $J$ values are lower, and the $J^{*}$ measure decidedly increases the co-occurrence coefficients.

Flares are our solar system's largest explosive events and they produce high energy particles and radiation that are dangerous to living organisms. Empirical studies show that flares are associated with emerging flux [19, 24, 44]. Using the $J$ measure, many emerging flux and flare (EF-FL) co-occurrences can be unfairly assessed as insignificant as they have small volumes. 

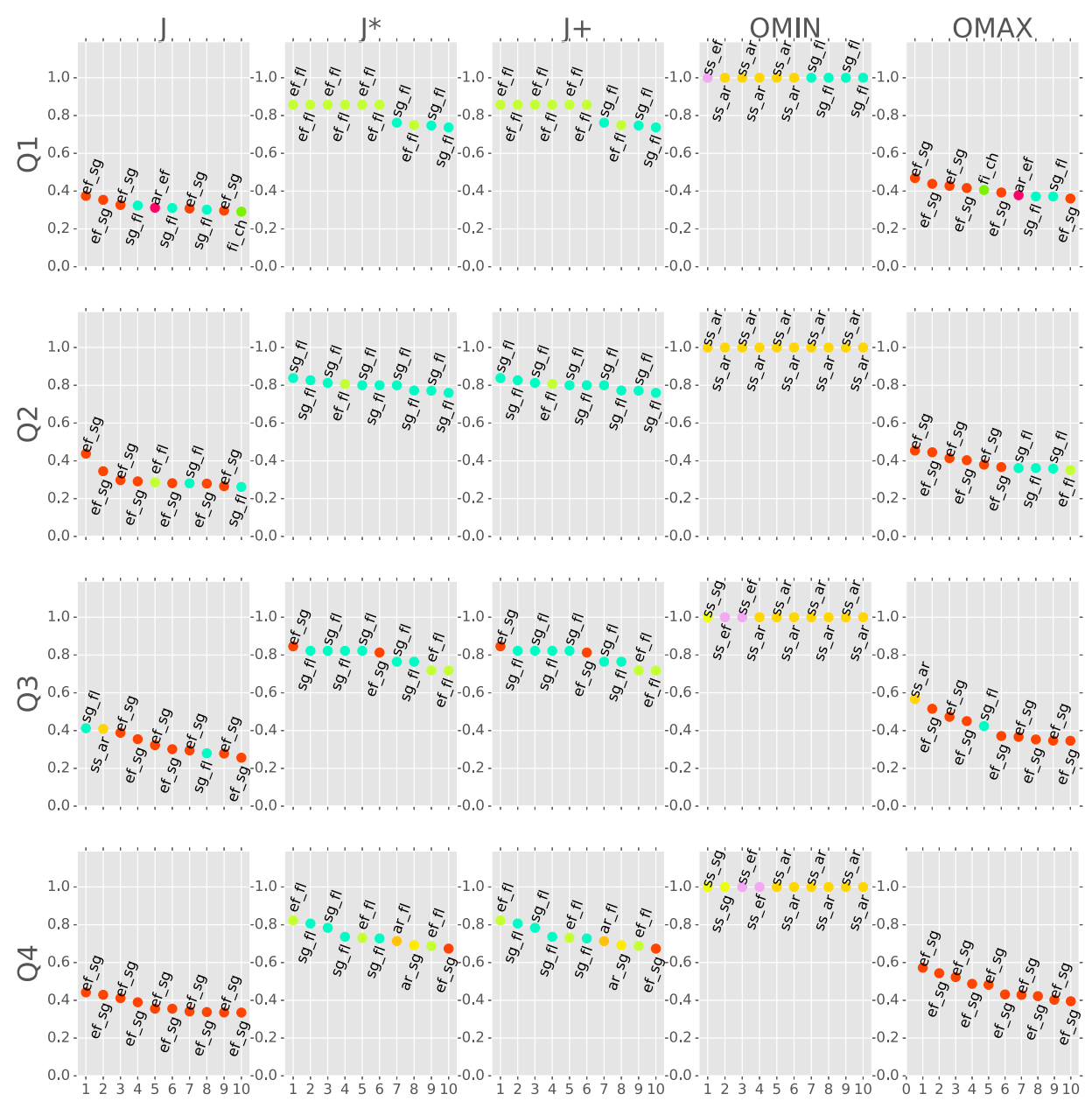

Fig. 10. Top-10 most significant individual spatiotemporal co-occurrences based on $J, J^{+}, J^{*}, O M I N$, and $O M A X$ measures with their specific spatiotemporal co-occurrence pattern type.

The $J^{*}$ measure can mitigate these problems, and can help find more relevant spatiotemporal co-occurrences.

6.2.4 Analysis of Top-10 Spatiotemporal Co-occurrences. In Figure 10, we demonstrate the top10 most significant spatiotemporal co-occurrences from the real-life solar datasets based on $J$, $J^{*}, J^{+}, O M A X$, and OMIN measures. For the OMIN measure, more than 10 co-occurrences are found to be having the maximal value of 1.0. Hence, the OMIN column shows a partial list of co-occurrences. The full list of all the co-occurrences and their significance assessments with our measures can be found on our project website [2].

Among quarterly datasets (Q1, Q2, Q3, and Q4), we see that our measures give similar top-10 most significant co-occurrence lists. For instance, the $J$ and $O M A X$ lists predominantly have EF-SG co-occurrences, while $J^{*}$ and $J^{+}$lists have at least two SG-FL and two EF-FL co-occurrences. This demonstrates that our results are consistent across different datasets. We also observe that the $J$ and OMAX measures mostly share their top-10 lists in all our datasets. We have also shown the 
correlation between the $J$ and $O M A X$ in Figure 6. On the other hand, the top-10 most significant co-occurrences based on $J^{+}$and $J^{*}$ are the same ones, because we found that the top-10 most significant co-occurrences from all of our datasets are size-2, and $J^{+}$and $J^{*}$ measures output the same significance value for size- 2 co-occurrences. Additionally, all the demonstrated spatiotemporal cooccurrences in Figure 10 are qualitatively well-known patterns, although there are no longitudinal quantitative studies that we know of on any of them.

Across different measures, in the top-10 most significant co-occurrence lists, we generally see EF-SG, EF-FL, SG-FL, and SS-AR co-occurrences. These are well-known patterns originating from active regions on the Sun. For instance, the emergence of magnetic flux is responsible for the formation of SS [45]. The EF tubes bring a large amount of magnetic energy to the solar surface. Extremely twisted flux tubes carry the most magnetic energy, and tend to give birth to very complex AR, which are anticipated to produce large FL.

It is generally accepted in the solar physics community that emerging flux, the formation of filament and sigmoids, and the occurrence of flares and CMEs are physically closely connected [49]. An alternative viewpoint to EF directly causing flares, CMEs, and sigmoids, as presented in [57] and [38], is that the newly emerged flux bumps into pre-existing magnetic field and leads to flux cancellation at the interface, a process directly leading to the formation of filaments, sigmoids, and flares as discussed in [39].

Another example relationship is the association between sigmoids and eruptions. The SG are the S-shaped structures in the surface of the Sun, and are known to be a good precursor of a number of solar eruptions such as flares and CMEs $[13,51]$. The correlation between EF and the formation of sigmoids is also widely known [12].

\subsection{Efficiency Analysis}

In this part of our evaluation, we discuss the running time requirements of the $J, J^{*}, J^{+}, O M A X$, and $O M I N$ measures for different types of co-occurrences. In Figures 11 and 12, we demonstrate the boxplots of running times of the measures for individual spatiotemporal co-occurrences among the instances of different event types. For brevity, we show the results from the Q1 solar event dataset. The results from Q2, Q3, and Q4 datasets are presented in the Online Appendix (Part D) of this article.

6.3.1 Implementation Details. The $J^{*}$ calculation algorithms are presented in Algorithms 1 and 2. The algorithms for $J, J^{+}, O M A X$, and $O M I N$ measures are presented in the Online Appendix (Part B). In our experimental runs, we stored the spatiotemporal instances in main memory. Our running time comparison solely includes the time required for procedures to calculate values of measures from given instances.

For providing a fair comparison, we implemented the measures in a similar fashion. We first find the intersection volumes, then union $\left(J, J^{*}\right.$, and $\left.J^{+}\right)$, minimum $(O M I N)$, or maximum $(O M A X)$ volumes. All the experiments are repeated five times for both artificial and solar event datasets, and average running times are reported.

6.3.2 Comparison of Running Times for Size-2 Co-occurrences. In Figure 11, the boxplots of running times for size-2 co-occurrences in the Q1 dataset are demonstrated. The running times are grouped by the event types of instances involved in the co-occurrences. Outliers are not demonstrated. Mean values are demonstrated with magenta dots, and median values are shown as red lines inside the boxes.

It can be observed from Figure 11 that for size- 2 co-occurrences, the $J^{*}$ and $J^{+}$calculations require less running time when compared to the $J$ calculations for all different types of events. As expected, $J^{*}$ and $J^{+}$measures, and OMAX and OMIN measures have very similar running 


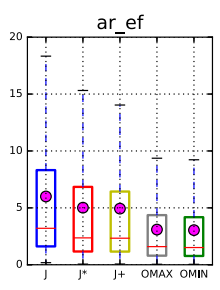

ef_ch

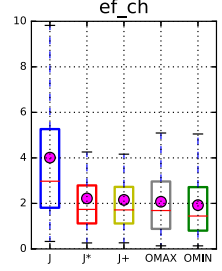

ss_fi

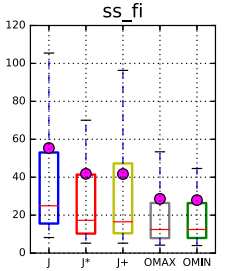

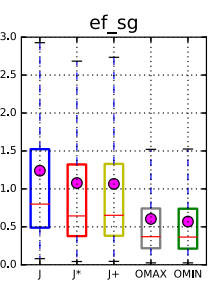

SS_fl

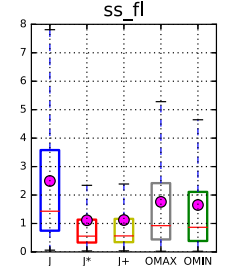

ar_sg

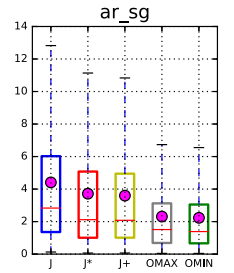

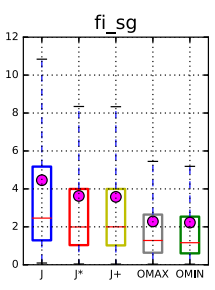

ar_fi

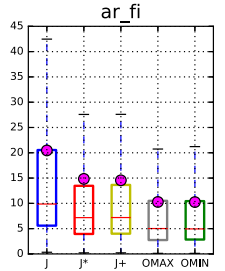

fi_fl

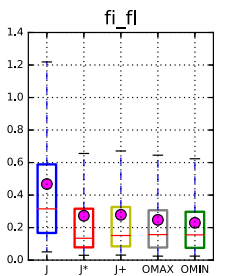

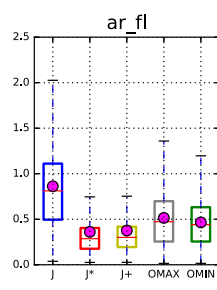

ss_ar

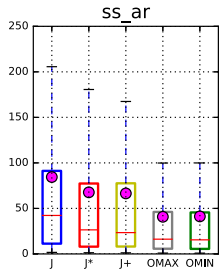

Ss_sg

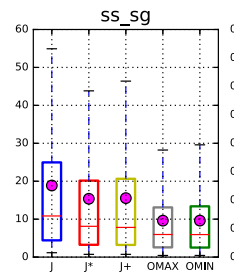

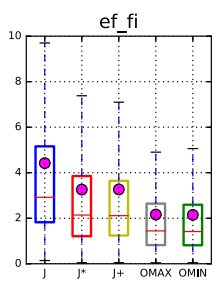

fi_ch

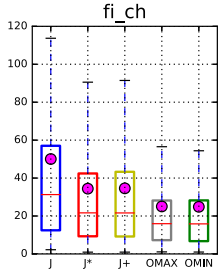

ef_fl

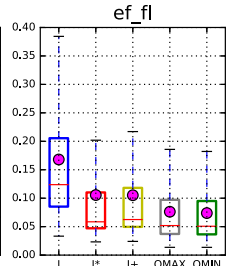

sg_fl

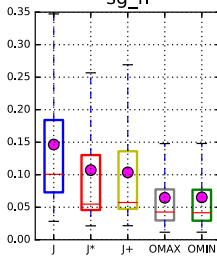

Ss_ef

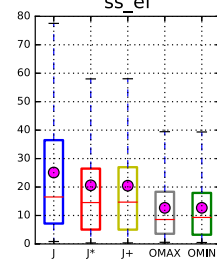

$\mathrm{Ch}_{-} \mathrm{fl}$

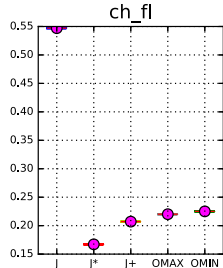

Fig. 11. The boxplots showing the running times (in milliseconds) for $J, J^{+}, J^{*}, O M I N$, and OMAX in size-2 co-occurrences in the Q1 dataset.

times. The longer running times are reported for pairs of long lifespan event instances (such as AR, SS, FI, and CH instances). Specifically, AR-SS pairs have the most computationally expensive calculations for all the measures. On the other hand, the co-occurrences involving short lifespan instances (such as EF, FL, and SG instances) require significantly less time. This situation is more noticeable when SS-AR and AR-FL co-occurrences are compared. AR-FL co-occurrences require two orders of magnitude less running times for measure calculations than SS-AR co-occurrences. This is an anticipated result because when measures are calculated the intersection volumes (and union volumes for $J^{*}$ and $J^{+}$) are determined by filtering the coexistence time intervals, and the filtering operation significantly reduces the computational load for short lifespan instances.

6.3.3 Comparison of Running Times for Size-3 Co-occurrences. In Figure 12, the boxplots of running times for size-3 co-occurrences are demonstrated. Similar to Figure 11, the running times for the measures are grouped together based on different event type triples. Note that not all the event types co-occur with each other. For instance, we did not identify any co-occurrences of AR-CH-SG or CH-EF-SS instances in the Q1 dataset.

From Figure 12, we can observe that average running time (mean) for OMAX and OMIN values are very close to each other. Among all five measures, the $J^{+}$is the most efficient one. For many size- 3 co-occurrences, the $J^{*}$ is more efficient than the $J$ measure. The $J$ is more efficient than $J^{*}$ for SS-AR-SG, SS-AR-FI, SS-AR-EF, and SS-AR-FL co-occurrences. This can be explained by the long co-occurrence time intervals between SS and AR instances. For many of the size-3 co-occurrences that include SS and AR instances, the cross co-occurrence time intervals are very long (as sunspots occur inside the active regions), which creates an overhead for $J^{*}$ calculations.

Similar to size-2 co-occurrences, the shortest calculation times are observed for co-occurrences among short lifespan instances such as EF, SG, or FL. See the boxplots for EF-SG-FL, FI-SG-FL, and 

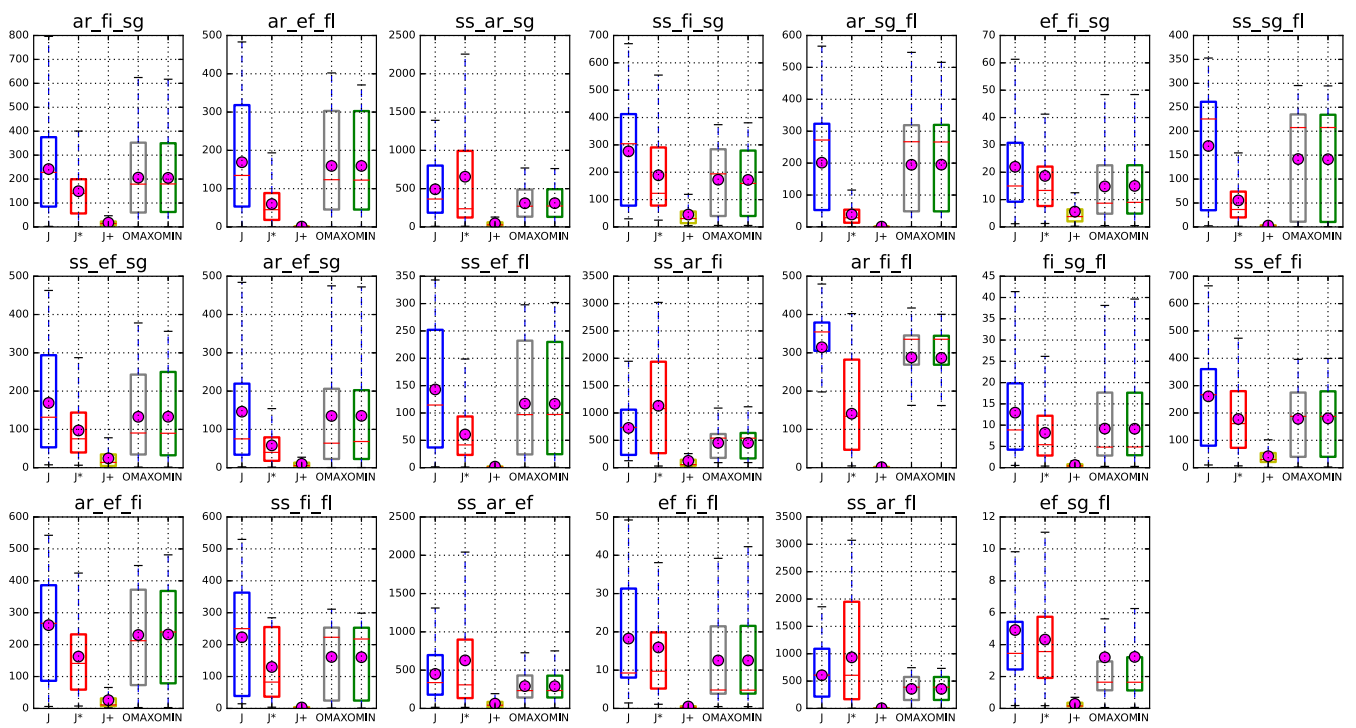

Fig. 12. The boxplots showing the running times (in milliseconds) for $J, J^{+}, J^{*}, O M I N$, and $O M A X$ in size-2 co-occurrences in the Q1 dataset.
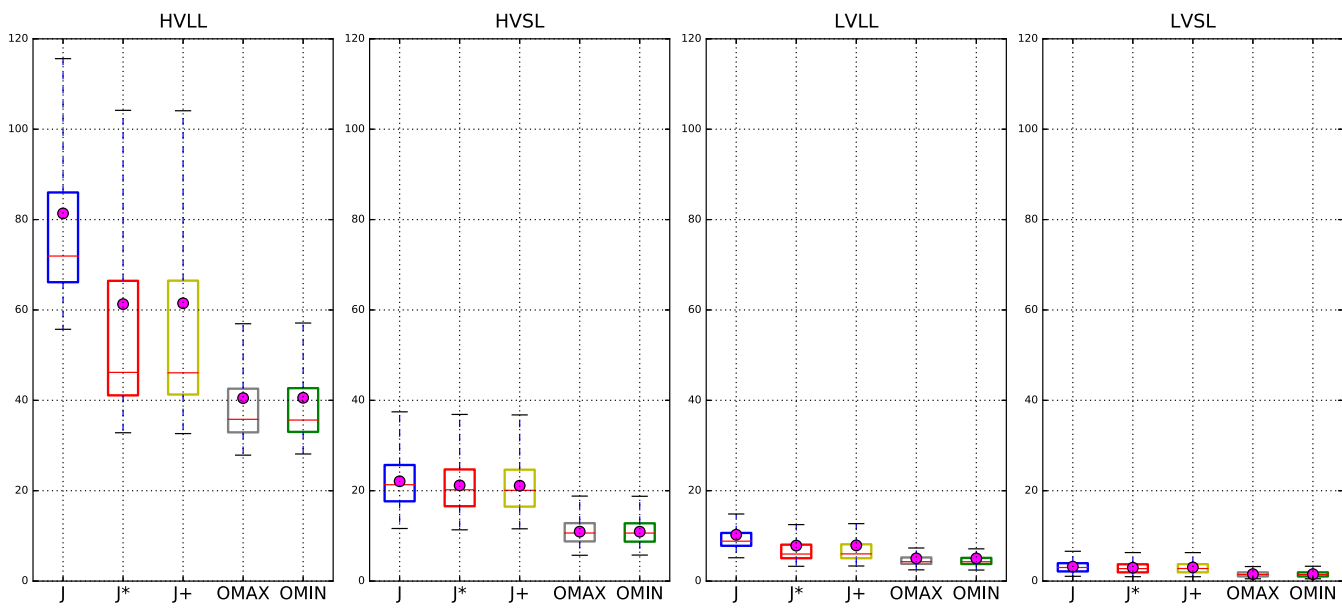

Fig. 13. The boxplots showing the running times for $J, J^{+}, J^{*}, O M I N$, and $O M A X$ in artificial datasets.

EF-FI-FL in Figure 12. The measure calculation times are significantly higher for co-occurrences between long lifespan instances. Particularly for SS-AR-FI co-occurrences, the average $J^{*}$ calculation time is greater than $1,000 \mathrm{~ms}$, while the mean $J^{*}$ calculation time for EF-SG-FL is $4 \mathrm{~ms}$.

6.3.4 Comparison of Running Times for Artificial Datasets. In Figure 13, the boxplots of running times for artificial datasets (HVLL, HVSL, $L V L L$, and $L V S L$ ) are demonstrated. All the artificial datasets include two artificial event types, each having 1,000 instances. The area values for the region polygons in the artificial datasets are the same. However, for observing the effect of spatial operations (i.e., union, intersection, intersects, and area), we change the number of vertices to create more complex region geometries. $L V_{-}$and $H V_{-}$prefixes denote low and high vertex counts 
in the region geometries represented as polygons. On the other hand, we altered the lifespans of the instances to observe the impact of temporal complexity._SL and _LL suffixes denote short and long lifespans of the instances.

From Figure 13, we can make following observations. In short lifespan datasets (LVSL and HVSL), the running times of $J$ are closer to $J^{*}$ 's. However, for long lifespan datasets (LVLL and HVLL), $J^{*}$ takes noticeably less time than $J$. Additionally, OMIN and OMAX are the most efficient measures for artificial datasets. Lastly, as expected, the spatially more complex HVSL (with short lifespans) dataset requires more running time for all the measures when compared to the temporally complex dataset LVLL (with low vertex counts).

6.3.5 Remarks on Running Times. From the running time analysis of size- 2 and size- 3 cooccurrences from solar event datasets (Figures 11 and 12) and artificial datasets (Figure 13), we can outline our remarks as follows:

- The OMIN and OMAX are the most computationally efficient measures for size-2 cooccurrences.

- The $J^{+}$is the most efficient measure for size-3 co-occurrences.

-For size-2 co-occurrences, the $J^{*}$ consistently takes less time than the $J$.

-For size- 3 co-occurrences, the $J^{*}$ usually takes less time than the $J$. In four types of cooccurrences involving long lifespan SS and AR instances, the $J$ calculation takes less time on average.

-Increasing spatial complexity (more complex polygons) impacts the running time more than increasing the temporal complexity (longer lifespans).

The main difference between the $J, J^{+}$, and $J^{*}$ calculation procedures is the identification of cross co-occurrence or co-occurrence time intervals. The $J^{*}$ calculation procedure for size- 2 cooccurrences has a shortcut for direct identification of cross co-occurrence time intervals. For larger size co-occurrences, the cross co-occurrence time intervals are found by examining the co-occurrence time intervals of each instance pair. This operation is expensive as it essentially checks whether a spatial overlap occurs between each geometry of each instance pair. On the other hand, the $\mathrm{J}^{+}$calculation is relatively less complex. The co-occurrence time intervals are found using a temporal coexistence filter, which reduces the search space. Although determining the co-occurrence or cross co-occurrence time intervals creates overhead, the $J^{+}$and $J^{*}$ measures calculate the union volume for only co-occurrence and cross co-occurrence time intervals, and remaining time intervals are not considered. Contrarily, the $J$ measure includes the union volumes of all instances.

In summary, for larger size co-occurrences, $J^{*}$ calculation can be less efficient as it needs to initially identify the cross co-occurrence time intervals. For spatiotemporal frequent pattern mining applications (such as STCOP mining [4]), initial identification of cross co-occurrence time intervals is not usually performed, as the larger size co-occurrences are found by using smaller size co-occurrences, and the cross co-occurrence time intervals can be easily identified. On the other hand, the $J^{*}$ can be more efficient for co-occurrences with certain characteristics (such as long lifespan instance vs. short lifespan instance; see running times of FI-AR-EF in Figure 12), as it only calculates the union volumes at cross co-occurrence time intervals.

\subsection{Suitability for STCOP Mining}

In this part of the experiments, we will discuss the measures from the perspective of STCOP mining. It should be noted that STCOP mining algorithms require antimonotonic measures for the correctness. Therefore, OMIN and $\mathrm{J}^{+}$measures, which do not an carry antimonotonic property 

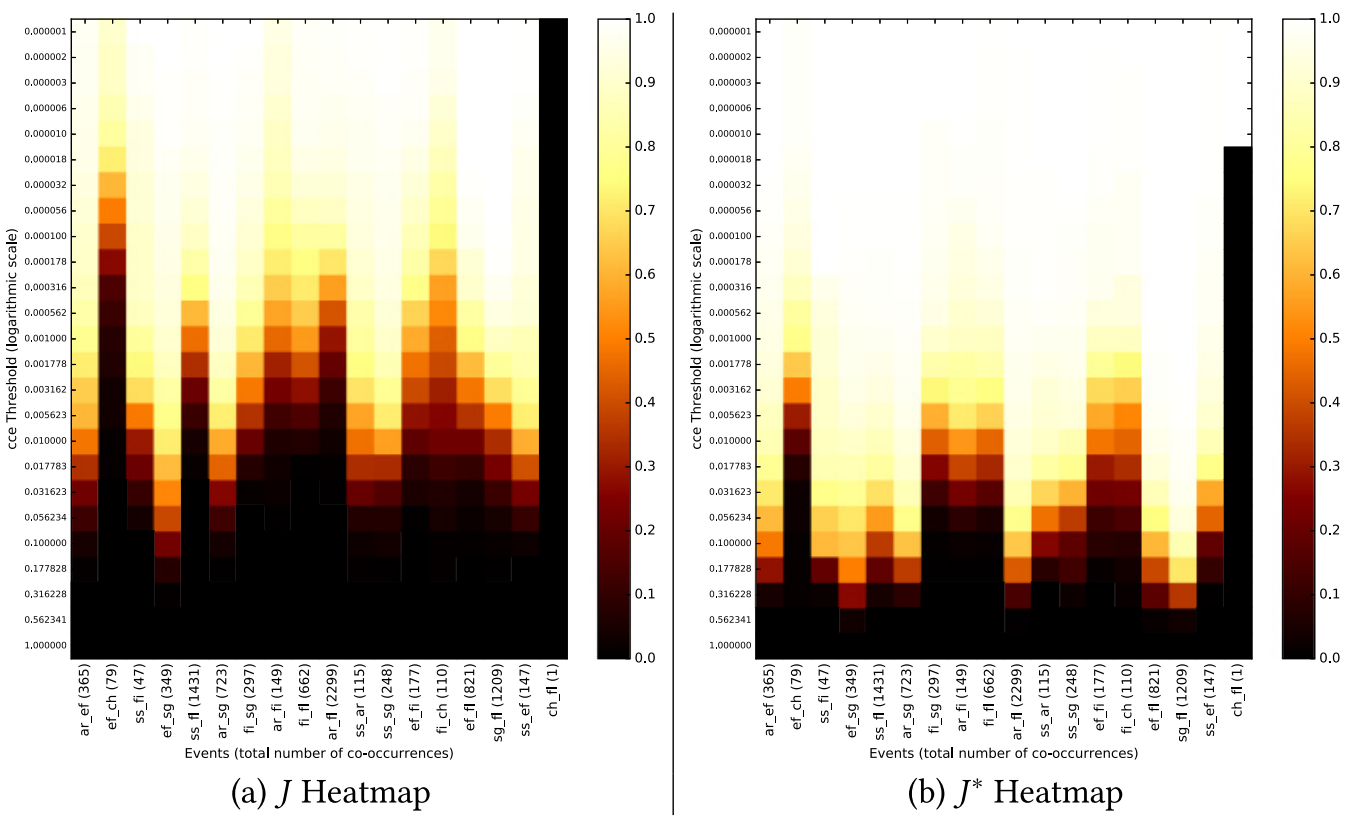

(b) $J^{*}$ Heatmap

Fig. 14. Heatmaps of the $J$ (in (a)) and $J^{*}$ (in (b)) values of size-2 co-occurrences for the Q1 dataset. The heatmaps demonstrate the ratio of the significant size-2 co-occurrences for different co-occurrence coefficient (cce) thresholds and event types of co-occurring instances, on $\mathrm{x}$ and $\mathrm{y}$ axes, respectively.

cannot be used in the context of current STCOP mining algorithms. We also showed that OMAX and $J$ values are very similar in Section 6.2. Therefore, here, we will compare the $J$ measure, which is currently used for ultimately determining the significance of co-occurrences, with our newly proposed $J^{*}$ measure.

In Figures 14 and 15, the heatmaps of $J$ and $J^{*}$ values for size- 2 and size-3 co-occurrences are demonstrated. Essentially, all the heatmaps encode information regarding the ratio of significant co-occurrences to all discovered co-occurrences (of particular event types) for specific co-occurrence coefficient (cce) threshold values. In Figures 14 and 15, the threshold values exponentially decrease from 1.0 to $0.000001\left(10^{-6}\right)$ by $\sqrt[4]{10}$.

In STCOP mining, a key challenge is to determine a meaningful co-occurrence coefficient threshold for the recognition of strong co-occurrences [10]. The co-occurrence coefficient threshold essentially implies the level of significance for the STCOP mining scheme. In other words, for a particular cce threshold, the STCOP mining algorithm identifies the patterns whose instances have a strong co-occurrence based on that cce threshold. Apart from the anomalies of $J$ measure in Section 6.2, for the spatiotemporal instances with highly unbalanced characteristics (e.g., solar event datasets), it is difficult to determine a threshold or a set of thresholds using the $J$ measure for STCOP mining analysis. The reason for that is the fluctuation of $J$ values happens in a very limited interval. We can observe from Figure 14 that for the $J$ values, the variability on the ratio of significant co-occurrences can only be observed in threshold interval from 0.1 to 0.0001 . Conversely, much of the variability for $J^{*}$ can be observed in the threshold interval from $\sim 0.316$ to 0.001 .

Another problem with using the $J$ is the exclusion of important co-occurrences or the inclusion of spurious co-occurrences. We have mentioned the strong association between flares and 


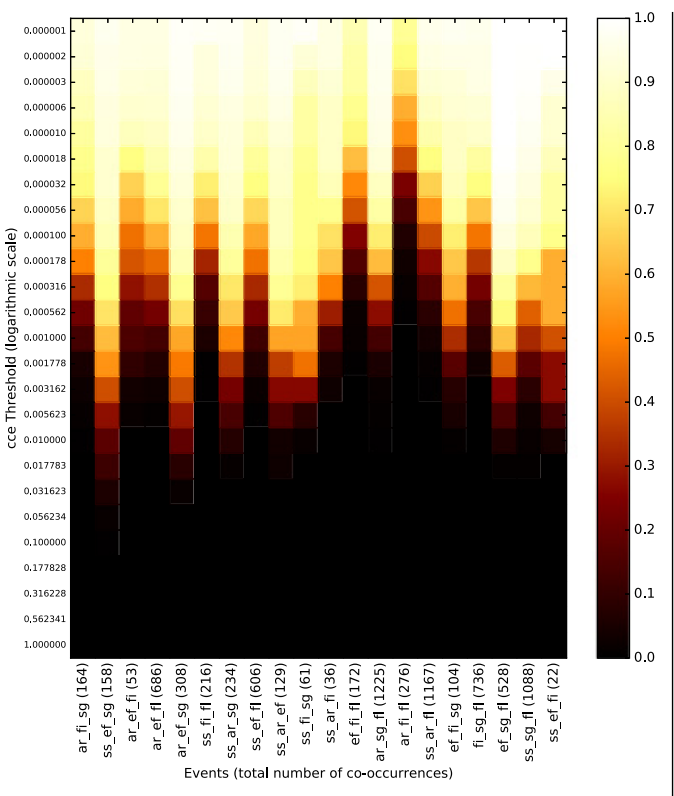

(a) $J$ Heatmap

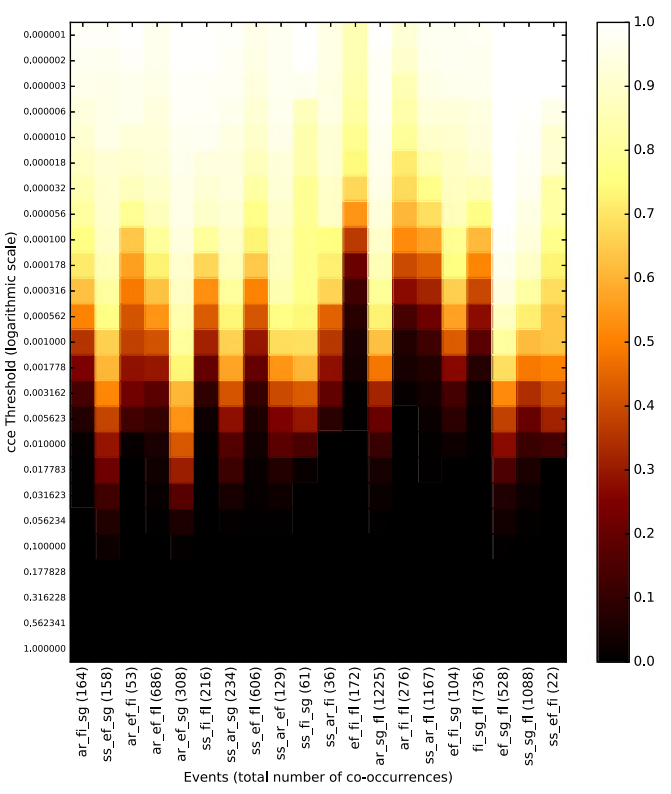

(b) $J^{*}$ Heatmap

Fig. 15. Heatmaps of the $J$ (in (a)) and $J^{*}$ (in (b)) values size-3 co-occurrences for the Q1 dataset. The heatmaps demonstrate the ratio of the significant size-3 co-occurrences for different co-occurrence coefficient (cce) thresholds and event types of co-occurring instances, on $\mathrm{x}$ and $\mathrm{y}$ axes, respectively.

active regions in Relevancy Analysis (Section 6.2). For including a very optimistic $30 \%$ of AR-FL co-occurrences in the Q1 dataset, the cce threshold should be set to less than 0.000562 . Such a mining schema would consider almost all the identified co-occurrences as significant, which makes the significance assessment procedures pointless because it would include all the co-occurrences including possibly spurious ones. Conversely, using the $J^{*}$ measure in STCOP mining, with a cce threshold set to 0.1 or 0.0562 , would include $60 \%$ to $80 \%$ of AR-FL co-occurrences, while preserving variations among the other co-occurrences.

\section{CONCLUSION}

In this article, we have presented two novel significance measures, $J^{+}$and $J^{*}$, which are specifically designed for determining the strength of spatiotemporal co-occurrences appearing among event instances. We have initially presented the shortcomings of the currently used OMAX and $J$ measures with example anomaly scenarios that can appear among the spatiotemporal co-occurrences. These anomalies can lead to unfair significance assessments that can impact the applicability of data mining algorithms to real-life datasets. As a solution to these anomalies, we introduced the $J^{+}$ and $J^{*}$ measures. Both $J^{+}$and $J^{*}$ are extensions to the $J$ measure. Our measures limit the volume calculations to specific regions of interests that are co-occurrence (for $\mathrm{J}^{+}$) and cross co-occurrence (for $J^{*}$ ) time intervals. We have also presented novel algorithms for the $J^{+}$and $J^{*}$ value calculations, which use temporal coexistence filtering. We have provided proofs for the antimonotonicity and containment properties of $J^{*}$. For demonstrating the effects of using our new measures for assessing the strength of co-occurrences, we have conducted our experiments with four solar event datasets and four artificial datasets. In our experiments, we have compared our measures with $J$, $O M I N$, and OMAX measures, and shown that $J^{*}$ and $J^{+}$can solve the anomalies created by the $J$ 
and OMAX measures. As a result of our experiments, we have confirmed that the $J^{*}$ measure is more efficient than $J$ measures, and can be utilized for discovering more meaningful spatiotemporal co-occurrences.

Our main future work lies in the analysis for automatically determining co-occurrence coefficient thresholds for mining statistically significant spatiotemporal co-occurrence patterns. Another future work is the design of efficient parallel algorithms for $J^{+}$and $J^{*}$ calculations.

\section{REFERENCES}

[1] Rakesh Agrawal, Tomasz Imielinski, and Arun N. Swami. 1993. Mining association rules between sets of items in large databases. In Proceedings of the 1993 ACM SIGMOD Interntional Conference on Management of Data 207-216. DOI : http://dx.doi.org/10.1145/170036.170072

[2] Berkay Aydin. 2016. Measuring the Significance of Spatiotemporal Co-occurrences. Retrieved August 26, 2017 from http://www.cs.gsu.edu/ baydin2/proj/jstar.html.

[3] Berkay Aydin, Vijay Akkineni, and Rafal Angryk. 2016. Modeling and indexing spatiotemporal trajectory data in non-relational databases. In Managing Big Data in Cloud Computing Environments. IGI Global, 133-162. DOI: http://dx.doi.org/doi:10.4018/978-1-4666-9834-5.ch006

[4] Berkay Aydin, Vijay Akkineni, and Rafal A. Angryk. 2015. Time-efficient significance measure for discovering spatiotemporal co-occurrences from data with unbalanced characteristics. In Proceedings of the 23rd SIGSPATIAL International Conference on Advances in Geographic Information Systems. 80:1-80:4. DOI : http://dx.doi.org/10.1145/2820783. 2820871

[5] Berkay Aydin and Rafal A. Angryk. 2015. Spatiotemporal frequent pattern mining on solar data: Current algorithms and future directions. In IEEE International Conference on Data Mining Workshop (ICDMW'15). 575-581. DOI: http://dx.doi.org/10.1109/ICDMW.2015.10

[6] Berkay Aydin and Rafal A. Angryk. 2016. Discovering spatiotemporal event sequences. In Proceedings of the 5th ACM SIGSPATIAL International Workshop on Mobile Geographic Information Systems (MobiGIS)16). 46-55. http://dl.acm.org/ citation.cfm?id=3004735.

[7] Berkay Aydin and Rafal A. Angryk. 2016. A graph-based approach to spatiotemporal event sequence mining. In IEEE International Conference on Data Mining Workshops (ICDM Workshops'16).1090-1097. DOI: http://dx.doi.org/10.1109/ ICDMW.2016.0157

[8] Berkay Aydin and Rafal A. Angryk. 2016. Spatiotemporal event sequence mining from evolving regions. In Proceedings of the 23rd International Conference on Pattern Recognition (ICPR'16). 4172-4177. DOI : http://dx.doi.org/10.1109/ICPR 2016.7900288

[9] Berkay Aydin, Rafal A. Angryk, and Karthik Ganesan Pillai. 2014. ERMO-DG: Evolving region moving object dataset generator. In Proceedings of the 27th FLAIRS International Conference. AAAI Press, 321-326. https://www.aaai.org/ ocs/index.php/FLAIRS/FLAIRS14/paper/view/7876.

[10] Berkay Aydin, Dustin Kempton, Vijay Akkineni, Shaktidhar Reddy Gopavaram, Karthik Ganesan Pillai, and Rafal A. Angryk. 2014. Spatiotemporal indexing techniques for efficiently mining spatiotemporal co-occurrence patterns. In Proceedings of the 2014 IEEE International Conference on Big Data (Big Data'14). 1-10. DOI : http://dx.doi.org/10.1109/ BigData.2014.7004398

[11] Roberto J. Bayardo, Jr. and Rakesh Agrawal. 1999. Mining the most interesting rules. In Proceedings of the 5th ACM SIGKDD International Conference on Knowledge Discovery and Data Mining (KDD'99). ACM, New York, 145-154. DOI : http://dx.doi.org/10.1145/312129.312219

[12] Richard C. Canfield, Hugh S. Hudson, and David E. McKenzie. 1999. Sigmoidal morphology and eruptive solar activity. Geophysical Research Letters 26, 6 (1999), 627-630. DOI : http://dx.doi.org/10.1029/1999GL900105

[13] Richard C. Canfield, Hugh S. Hudson, and Alexei A. Pevtsov. 2000. Sigmoids as precursors of solar eruptions. IEEE Transactions on Plasma Science 28, 6 (2000), 1786-1794. DOI : http://dx.doi.org/10.1109/27.902208

[14] Huiping Cao, Nikos Mamoulis, and David W. Cheung. 2006. Discovery of collocation episodes in spatiotemporal data. In Proceedings of the 6th IEEE International Conference on Data Mining (ICDM'06). 823-827. DOI : http://dx.doi.org/10. 1109/ICDM.2006.59

[15] Mete Celik. 2015. Partial spatio-temporal co-occurrence pattern mining. Knowledge and Information Systems 44, 1 (Jul 2015), 27-49. DOI : http://dx.doi.org/10.1007/s10115-014-0750-2

[16] Mete Celik, Shashi Shekhar, James P. Rogers, James A. Shine, and Jin Soung Yoo. 2006. Mixed-drove spatio-temporal co-occurence pattern mining: A summary of results. In Proceedings of the 6th IEEE International Conference on Data Mining (ICDM'06). 119-128. DOI : http://dx.doi.org/10.1109/TKDE.2008.97

[17] Elena Deza and Michel-Marie Deza. 2006. General definitions. In Dictionary of Distances, Elena Dezaand Michel-Marie Deza (Eds.). Elsevier, Amsterdam, 2-30. DOI : http://dx.doi.org/10.1016/B978-044452087-6/50001-9 
[18] Martin Ester, Hans-Peter Kriegel, Jörg Sander, and Xiaowei Xu. 1996. A density-based algorithm for discovering clusters in large spatial databases with noise. In Proceedings of the 2nd International Conference on Knowledge Discovery and Data Mining (KDD'96). 226-231. http://dl.acm.org/citation.cfm?id=3001460.3001507

[19] Joan Feynman and Sara F. Martin. 1995. The initiation of coronal mass ejections by newly emerging magnetic flux. Journal of Geophysical Research: Space Physics 100, A3 (1995), 3355-3367. DOI : http://dx.doi.org/10.1029/94JA02591

[20] Soukaina Filali Boubrahimi, Berkay Aydin, Dustin Kempton, and Rafal A. Angryk. 2016. Spatio-temporal interpolation methods for solar events metadata. In Proceedings of the 2016 IEEE International Conference on Big Data (BigData'16). 3149-3157. DOI : http://dx.doi.org/10.1109/BigData.2016.7840970

[21] Liqiang Geng and Howard J. Hamilton. 2006. Interestingness measures for data mining: A survey. ACM Computing Surveys 38, 3 (2006). DOI : http://dx.doi.org/10.1145/1132960.1132963

[22] Joachim Gudmundsson, Marc J. van Kreveld, and Bettina Speckmann. 2004. Efficient detection of motion patterns in spatio-temporal data sets. In Proceedings of the 12th ACM International Workshop on Geographic Information Systems (ACM-GIS'04). 250-257. DOI : http://dx.doi.org/10.1145/1032222.1032259

[23] Jiawei Han, Jian Pei, and Yiwen Yin. 2000. Mining frequent patterns without candidate generation. In Proceedings of the 2000 ACM SIGMOD International Conference on Management of Data1-12. DOI : http://dx.doi.org/10.1145/335191. 335372

[24] Jean Heyvaerts, Eric R. Priest, and David M. Rust. 1977. An emerging flux model for the solar flare phenomenon. The Astrophysical fournal 216 (1977), 123-137. DOI : http://dx.doi.org/10.1086/155453

[25] Jochen Hipp, Ulrich Güntzer, and Gholamreza Nakhaeizadeh. 2000. Algorithms for association rule mining-A general survey and comparison. SIGKDD Explorations 2, 1 (2000), 58-64. DOI : http://dx.doi.org/10.1145/360402.360421

[26] M. Shahriar Hossain and Rafal A. Angryk. 2007. GDClust: A graph-based document clustering technique. In Proceedings of the 7th IEEE International Conference on Data Mining Workshops (ICDM'07). 417-422. DOI : http://dx.doi.org/ 10.1109/ICDMW.2007.104

[27] Yan Huang, Jian Pei, and Hui Xiong. 2006. Mining co-location patterns with rare events from spatial data sets. GeoInformatica 10, 3 (2006), 239-260. DOI : http://dx.doi.org/10.1007/s10707-006-9827-8

[28] Yan Huang, Shashi Shekhar, and Hui Xiong. 2004. Discovering colocation patterns from spatial data sets: A general approach. IEEE Transactions on Knowledge and Data Engineering 16, 12 (2004), 1472-1485.

[29] N. Hurlburt, M. Cheung, C. Schrijver, L. Chang, S. Freeland, S. Green, C. Heck, A. Jaffey, A. Kobashi, D. Schiff, J. Serafin, R. Seguin, G. Slater, A. Somani, and R. Timmons. 2010. Heliophysics event knowledgebase for the solar dynamics observatory (SDO) and beyond. Solar Physics 275, 1 (2010), 67-78. DOI : http://dx.doi.org/10.1007/s11207-010-9624-2

[30] Panos Kalnis, Nikos Mamoulis, and Spiridon Bakiras. 2005. On discovering moving clusters in spatio-temporal data. In Proceedings of the 9th International Symposium on Advances in Spatial and Temporal Databases (SSTD'05). 364-381. DOI : http://dx.doi.org/10.1007/11535331_21

[31] Barry D. Keim, Robert A. Muller, and Gregory W. Stone. 2007. Spatiotemporal patterns and return periods of tropical storm and hurricane strikes from Texas to Maine. Journal of Climate 20, 14 (2007), 3498-3509.

[32] Dustin Kempton and Rafal A. Angryk. 2015. Tracking solar events through iterative refinement. Astronomy and Computing 13 (2015), 124-135. DOI : http://dx.doi.org/10.1016/j.ascom.2015.10.005

[33] Krzysztof Koperski and Jiawei Han. 1995. Discovery of spatial association rules in geographic information databases. In Advances in Proceedings of the 4th International Symposium on Spatial Databases (SSD'95). 47-66. http://dl.acm.org/ citation.cfm?id=647224.718925.

[34] Sam Krucker, Arnold O. Benz, Timothy S. Bastian, and Loren W. Acton. 1997. X-ray network flares of the quiet sun. The Astrophysical fournal 488 (Oct. 1997), 499-505. DOI : http://dx.doi.org/10.1086/304686

[35] Patrick Laube and Stephan Imfeld. 2002. Analyzing relative motion within groups of trackable moving point objects. In Proceedings of the 2nd International Conference on Geographic Information Science (GIScience'02). 132-144. DOI : http://dx.doi.org/10.1007/3-540-45799-2_10

[36] Patrick Laube, Marc van Kreveld, and Stephan Imfeld. 2005. Finding REMO-Detecting Relative Motion Patterns in Geospatial Lifelines. Springer, Berlin, 201-215. DOI : http://dx.doi.org/10.1007/3-540-26772-7_16

[37] LMSAL. 2014. Heliophysics Event Registry. Retrieved June 26, 2014 from http://lmsal.com/isolsearch.

[38] Petrus C. Martens and Cornelis Zwaan. 2001. Origin and evolution of filament-prominence systems. The Astrophysical fournal 558, 2 (2001), 872. https://doi.org/10.1086/322279.

[39] Sara F. Martin. 1998. Conditions for the formation and maintenance of filaments-(Invited review). Solar Physics 182, 1 (1998), 107-137. DOI : http://dx.doi.org/10.1023/A:1005026814076

[40] Earle. B. Mayfield and Jon K. Lawrence. 1985. The correlation of solar flare production with magnetic energy in active regions. Solar Physics 96 (April 1985), 293-305. DOI : http://dx.doi.org/10.1007/BF00149685

[41] Kenneth McGarry. 2005. A survey of interestingness measures for knowledge discovery. Knowledge Engineering Review 20, 1 (2005), 39-61. DOI : http://dx.doi.org/10.1017/S0269888905000408 
[42] Yasuhiko Morimoto. 2001. Mining frequent neighboring class sets in spatial databases. In Proceedings of the 7th ACM SIGKDD International Conference on Knowledge Discovery and Data Mining. 353-358. DOI : http://dx.doi.org/10.1145/ 502512.502564

[43] Micheal J. Murray, Lidia van Driel-Gesztelyi, and Deborah Baker. 2009. Simulations of emerging flux in a coronal hole: Oscillatory reconnection. Astronomy \& Astrophysics 494, 1 (2009), 329-337. DOI : http://dx.doi.org/10.1051/0004-6361: 200810406

[44] Nariaki V. Nitta and Hugh S. Hudson. 2001. Recurrent flare/CME events from an emerging flux region. Geophysical Research Letters 28, 19 (2001), 3801-3804. DOI : http://dx.doi.org/10.1029/2001GL013261

[45] Eugene N. Parker. 1979. Sunspots and the physics of magnetic flux tubes. I-The general nature of the sunspot. IIAerodynamic drag. The Astrophysical fournal 230 (1979), 905-923. DOI : http://dx.doi.org/10.1086/157150

[46] Karthik Ganesan Pillai, Rafal A. Angryk, and Berkay Aydin. 2013. A filter-and-refine approach to mine spatiotemporal co-occurrences. In Proceedings of the 21st SIGSPATIAL International Conference on Advances in Geographic Information Systems (SIGSPATIAL'13). 104-113. DOI : http://dx.doi.org/10.1145/2525314.2525367

[47] Karthik Ganesan Pillai, Rafal A. Angryk, Juan M. Banda, Michael A. Schuh, and Tim Wylie. 2012. Spatio-temporal cooccurrence pattern mining in data sets with evolving regions. In Proceedings of the 12th IEEE International Conference on Data Mining Workshops. 805-812. DOI : http://dx.doi.org/10.1109/ICDMW.2012.130

[48] Karthik Ganesan Pillai, Rafal A. Angryk, Juan M. Banda, Tim Wylie, and Michael A. Schuh. 2014. Spatiotemporal co-occurrence rules. In New Trends in Databases and Information Systems. Springer, 27-35. DOI : http://dx.doi.org/10. 1007/978-3-319-01863-8_3

[49] Eric Priest. 2014. Magnetohydrodynamics of the Sun. Cambridge University Press.

[50] Stéphane Régnier and Richard C. Canfield. 2006. Evolution of magnetic fields and energetics of flares in active region 8210. Astronomy \& Astrophysics 451, 1 (2006), 319-330. https://doi.org/10.1051/0004-6361:20054171.

[51] David M. Rust and Ashok Kumar. 1996. Evidence for helically kinked magnetic flux ropes in solar eruptions. The Astrophysical fournal Letters 464, 2 (1996), L199. DOI : http://dx.doi.org/10.1086/310118

[52] Space Studies Board. 2008. Severe Space Weather Events-Understanding Societal and Economic Impacts: A Workshop Report-Extended Summary. National Academies Press.

[53] Michael Steinbach and Vipin Kumar. 2007. Generalizing the notion of confidence. Knowledge and Information Systems 12, 3 (2007), 279-299. DOI : http://dx.doi.org/10.1109/ICDM.2005.72

[54] Pang-Ning Tan, Vipin Kumar, and Jaideep Srivastava. 2002. Selecting the right interestingness measure for association patterns. In Proceedings of the 8th ACM SIGKDD International Confeerence on Knowledge Discovery and Data Mining. 32-41. DOI : http://dx.doi.org/10.1145/775047.775053

[55] Pang-Ning Tan, Michael Steinbach, and Vipin Kumar. 2005. Introduction to Data Mining. Addison-Wesley.

[56] Peter John Taylor. 1977. Quantitative Methods in Geography: An Introduction to Spatial Analysis. Houghton Mifflin, Boston.

[57] Adrian A. van Ballegooijen and Petrus. C. Martens. 1989. Formation and eruption of solar prominences. The Astrophysical Journal 343 (Aug. 1989), 971-984. DOI : http://dx.doi.org/10.1086/167766

[58] Cornelis J. van Rijsbergen. 1979. Information Retrieval. Butterworth.

[59] Hui Xiong, Shashi Shekhar, Yan Huang, Vipin Kumar, Xiaobin Ma, and Jin Soung Yoo. 2004. A framework for discovering co-location patterns in data sets with extended spatial objects. In Proceedings of the 4th SIAM International Conference on Data Mining. 78-89. https://doi.org/10.1137/1.9781611972740.8.

[60] Zeng-Jie Yang and Robert J. Wechsler-Reya. 2007. Hit'em where they live: Targeting the cancer stem cell niche. Cancer Cell 11, 1 (2007), 3-5. DOI : http://dx.doi.org/10.1016/j.ccr.2006.12.007

[61] Jin Soung Yoo, Shashi Shekhar, Sangho Kim, and Mete Celik. 2006. Discovery of co-evolving spatial event sets. In Proceedings of the 6th SIAM International Conference on Data Mining. 306-315. DOI: http://dx.doi.org/10.1137/1 9781611972764.27

Received April 2016; revised September 2017; accepted September 2017 